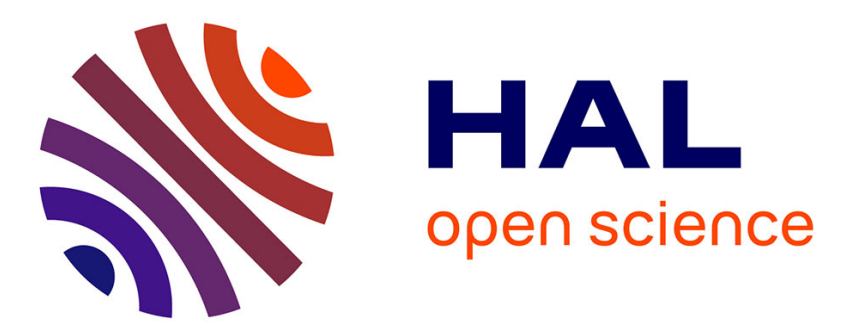

\title{
Studying the SINR process of the typical user in Poisson networks by using its factorial moment measures
}

Bartlomiej Blaszczyszyn, Holger Keeler

\section{To cite this version:}

Bartlomiej Blaszczyszyn, Holger Keeler. Studying the SINR process of the typical user in Poisson networks by using its factorial moment measures. IEEE Transactions on Information Theory, 2015, 61 (12), pp.6774 - 6794. 10.1109/TIT.2015.2484348 . hal-00932106v3

\section{HAL Id: hal-00932106 https://inria.hal.science/hal-00932106v3}

Submitted on 11 Mar 2015

HAL is a multi-disciplinary open access archive for the deposit and dissemination of scientific research documents, whether they are published or not. The documents may come from teaching and research institutions in France or abroad, or from public or private research centers.
L'archive ouverte pluridisciplinaire HAL, est destinée au dépôt et à la diffusion de documents scientifiques de niveau recherche, publiés ou non, émanant des établissements d'enseignement et de recherche français ou étrangers, des laboratoires publics ou privés. 


\title{
Studying the SINR process of the typical user in Poisson networks by using its factorial moment measures
}

\author{
Bartłomiej Błaszczyszyn* and Holger Paul Keeler* \\ *Inria-ENS, 23 Avenue d'Italie, 75214 Paris, FRANCE \\ Bartek.Blaszczyszyn@ens.fr, Holger.Keeler@inria.fr
}

\begin{abstract}
Based on a stationary Poisson point process, a wireless network model with random propagation effects (shadowing and/or fading) is considered in order to examine the process formed by the signal-to-interference-plus-noise ratio (SINR) values experienced by a typical user with respect to all base stations in the down-link channel. This SINR process is completely characterized by deriving its factorial moment measures, which involve numerically tractable, explicit integral expressions. This novel framework naturally leads to expressions for the $k$-coverage probability, including the case of random SINR threshold values considered in multi-tier network models. While the $k$-coverage probabilities correspond to the marginal distributions of the order statistics of the SINR process, a more general relation is presented connecting the factorial moment measures of the SINR process to the joint densities of these order statistics. This gives a way for calculating exact values of the coverage probabilities arising in a general scenario of signal combination and interference cancellation between base stations. The presented framework consisting of mathematical representations of SINR characteristics with respect to the factorial moment measures holds for the whole domain of SINR and is amenable to considerable model extension.
\end{abstract}

\section{Index Terms}

Heterogeneous networks, multi-tier networks, Poisson process, Poisson-Dirichlet process, SINR, coverage probabilities, interference cancellation, antenna cooperation, shadowing, fading, propagation invariance, factorial moment expansions, stochastic geometry.

\section{INTRODUCTION}

The steady rise of heterogeneous cellular networks, owing to the deployment of recent technologies such as femtocells and picocells to handle increased user-data, is driving the need for new and more robust analytic methods. Based on information theoretic arguments, a key performance metric is the signal-to-interference-plus-nose ratio (SINR) in the downlink channel experienced by a typical user in the network. The SINR (or without noise simply SIR) is a function of propagation processes, which incorporate the distance-dependent path-loss function and (often assumed to be random) fading and/or shadowing, which we refer to as simply propagation effects. Knowledge of the distribution of the SINR leads to the coverage probability and other SINR-based characteristics (such as e.g the spectral efficiency) of the cellular networks, which can be used to better design and implement such networks.

The irregularity of network base station locations suggests that their placement is often best assumed to be random. This claim has been supported in recent years with tractable stochastic geometry models based on the Poisson point process yielding accurate solutions [1]. In addition to the tractability and 'worst-case' arguments for Poisson models, recent convergence results $[2,3]$ has shown that a wide class of stationary network configurations give results for functions of propagation processes, such as the SINR, as though the placement of the base stations is a Poisson process when sufficiently strong shadowing is incorporated into the mode, which was previously predicted via simulations [4]. This adds weight to the argument for using the Poisson model, which leads to more tractable expressions for the SINR and related quantities.

Motivated by the ongoing deployment of heterogeneous networks and recent investigations for mathematical expressions of the distribution of the SINR in Poisson models of these and other networks (see related work in Section I-A), we wish to introduce a general framework for studying arbitrary functions of the process formed by the SINR values experienced by a typical user with respect to all base stations in the down-link channel. To meet this end we completely characterize this process by deriving explicit, numerically tractable integral expressions for its factorial moment measures of all orders $n \geq 1$. These measures represent the expected number of ways that the typical user can connect to $n$ different base stations at different SINR threshold values. It is known that various characteristics of an arbitrary point process admit series expansion representations involving integrals with respect to its factorial moment measures [5, 6]. Surprisingly, in the case of the SINR process many interesting characteristics (including $k$-coverage probability, coverage under signal combination and interference cancellation) can be written as expansions with finite number of terms, hence the expansions need not be approximations. This observation, reminiscent of the existence of the pole capacity in cellular networks, is a consequence of an algebraic property of the SINR 
process, limiting the maximum number of stations that can cover a given point with a SINR bounded away from zero. The calculation of the corresponding expansion terms is arguably more intuitive and computationally more efficient than previous methods based on inversions of Laplace transforms for studying similar problems.

The SINR process, considered above, is itself a function of the propagation process, already studied in cellular networks, and thus inherits the invariance/equivalence properties with respect to the distribution of the random propagation effects (fading and/or shadowing) [7]. In fact, we also consider a process formed by the values of the signal-to-total-interference-and-noise ratio (STINR). Working with this latter process is more convenient and a simple mapping allows one to interpret the results in terms of the original SINR process.

In summary, we define a point process formed by the SINR values experienced by a typical user with respect to all base stations in the down-link channel. The key result is the complete characterization of the distribution of this process via its factorial moment measures. These measures lead in a natural way to the finite-dimensional distributions of the order statistics of the SINR process thus allowing one to study general functions of several strongest values of the SINR process. We present particular probability results for $k$-coverage and coverage with signal combination and interference cancellation.

\section{A. Related work}

1) Propagation equivalence: In this work we employ a useful result, recently called propagation invariance by Błaszczyszyn and Keeler [7], that arises due to the model being based on a Poisson process and the path-loss function being a singular power-law. A classical and related result on the invariance of shot noise for many types of functions was discovered long ago by Gilbert and Pollak [8], and later examined in further detail by Lowen and Teich [9] for power-law functions, hence revealing that Poisson shot noise only depends one moment.

For wireless networks, Haenggi [10, Proposition 3] observed the so-called propagation invariance, as a particular case, and derived related results under a Nakagami fading model. In the context of SINR of cellular networks, Błaszczyszyn et al. [11] independently observed this invariance characteristic for interference and propagation losses in general, thus allowing propagation effects to be incorporated into the model by just one moment. Pinto et.al [12] derived and used a similar result to show that the node degree of secrecy graphs (based on Poisson processes) is invariant for the distribution of propagation effects. In all three papers [10-12], the invariance results are obtained by defining a point process on the positive real line, which we call the propagation (loss) process, and showing that if the base station configuration forms a homogeneous Poisson process, then the propagation process is an inhomogeneous Poisson point process on the positive real line. More specifically, Błaszczyszyn et al. [11, Proposition 5.5] observed that this propagation process depends on only one moment of the propagation effects, and not its distribution, by explicitly deriving its density measure. Conversely, Pinto et.al [12, Theorem 3.3] did not strictly observe propagation invariance in our sense as they used a more general path-loss function and not necessarily the singular power-law ${ }^{1}$, and proved their invariance result for another quantity by mapping different (inhomogeneous Poisson) propagation processes and obtaining an identical process. Haenggi [10] independently defined the propagation process (as "path loss process with fading") and observed [10, Proposition 1] that it is a (possibly inhomogeneous) Poisson process without stating its density measure in general form.

2) SINR coverage: Several authors recently developed and studied single and multi-tier network models ${ }^{2}$ based on the Poisson process that led to more or less closed-form expressions for the (downlink) SINR coverage probability of the typical user. In general, all these models assume that the serving base station(s) are those that somehow offer the strongest received signal power. However, they make different assumptions on what kind of propagation effects are taken into account when maximizing the received signal.

Dhillon et al. [13, 14], Madhusudhanan et al. [15, 16] and Mukherjee [17] assume that the selection is made on the instantaneous (possibly tier-biased) received power, i.e., when all the propagation effects are taken into account. In this case the actual distribution of the propagation effects does not matter (propagation invariance admitted in [15, 16]), and the results should be the same (up to constants multiplying the node densities) regardless whether the exponential shadowing-and-fading distribution is assumed for mathematical convenience $[13,14,17]$ or the expressions are developed using an equivalent network representation with no random propagation effects (the serving station is the closes one) [15, 16]. All these works observe that the analysis simplifies when the SINR (or SIR) threshold $\tau$, which is the technology-dependent level that the SINR must exceed to establish a connection, is greater than one (or zero in $\mathrm{dB}$ ). This assumption on the $\tau$ value (or multiple $\tau$ values in the multi-tier model) implies that at maximum only one base station in the entire network at any instant can cover the typical user. Mukherjee [17] goes beyond this assumption (considers $\tau<1$ ) using a variant of the inclusion-exclusion formula. This approach was used independently by Keeler at. al [18] to study the $k$-coverage in a single-tier network. Madhusudhanan et al. $[15,16]$ also consider $\tau<1$ but their results involve the inversion of Laplace transforms and hence are less explicit. Inversion of Laplace transforms is also exploited in [2].

\footnotetext{
${ }^{1}$ Under certain conditions, there exists a trade-off between how general the path-loss function can be and to which stochastic process the invariance result applies. Assuming a singular power-law path-loss function results in the invariance property applying to the propagation processes. The invariance result of Pinto et al. [12] applies to a function of propagation processes, hence it holds under more general path-loss functions in the spirit of Gilbert and Pollak [8].

${ }^{2}$ Terms "multi-tier" and "heterogeneous" networks are often used interchangeably, but we later adopt a model-specific terminology in which the former is an instance of the latter.
} 
A different class of models is considered in Mukherjee [19], Jo et al. [20], and also, for a single-tier network, in Vu et al. [21] and Keeler et al. [18, Section IV.C]. These models assume that some propagation effects (say rapidly changing fading) impact the SINR value but are not taken into account when choosing the strongest (serving) base station. In this case the coverage probability depends on the particular distribution of this fading and only Rayleigh case leads to closed form expressions. Moreover, in this case the assumption $\tau \geq 1$ is no longer crucial. This way of taking into account the fading (obviously) leads to smaller coverage probabilities and the difference is relatively important for smaller values of $\tau$, as illustrated in [18].

A combination of the two scenarios is considered in Mukherjee [22], where the Rayleigh fading is assumed and the serving base station is either the instantaneously the strongest one (when accounting for the fading) or the one nearest to the user (after accounting for cell selection bias).

For tractability, most of the aforementioned results assume constant path-loss exponents. Models with different (but constant) path-loss exponent on each tier are considered in [16, 19, 20,22]. Authors in [16] observe that a multi-tier network is stochastically equivalent to a single-tier network with unity parameters while all the original parameters are incorporated into the density of the (inhomogeneous Poisson) propagation process. This result was later extended in [7] to the case of random path-loss exponents and other parameters in a Poisson network, thus showing a random heterogeneous network is equivalent to a network with constant parameters and an isotropic base station density. This equivalence result, a variation of which we use here, allowed for the comparison of markedly different networks, for example single-tier and multi-tier types, by examining their equivalent (isotropic) forms.

3) Cooperation and interference cancellation: In this work we do not aim to perform detailed analysis on various types of cooperation or interference cancellation, but rather demonstrate the power of the factorial moment approach by deriving coverage probability for two proposed models of these respective schemes. That said, research featuring stochastic geometry models of cooperation or interference cancellation has remained relatively untouched until recently. Baccelli and Giovanidis [23] recently introduced a stochastic geometry framework for studying pair-wise base station cooperation in cellular networks. They make the tractability assumption of Rayleigh fading ${ }^{3}$ and use Laplace techniques to derive expressions for the coverage probability under geometry-based cooperation policies with a parameter (for optimization purposes) representing the degree of cooperation. Parallel to this work Akoum and Heath [24] developed a Poisson-based stochastic geometry model in order to examine interference coordination by introducing the concept of cooperation clusters. They assume Rayleigh fading and a non-singular power-law path-loss function and represent coordination clusters with a (Poisson-Poisson) cluster process, which leads to bounds on the coverage probability expression.

In the setting of interference cancellation, Zhang and Haenggi $[25,26]$ tackled the problem of calculating the probability of decoding and removing $n$ interfering signals in Poisson networks by independently using the aforementioned approach of mapping propagation effects and the path-loss values to the positive real line. Provided $\tau \geq 1$, they derived [26] a closed-form expression for the SIR-based probability of decoding the $n$-th strongest signal, and used it to obtain bounds for the mean number of decoded users and the probability of decoding $n$ users. Later Zhang and Haenggi [27] applied these results to a heterogeneous network model (with single $\tau$ ) and concluded that a large part of the performance gained from interference cancellation is possible by just removing the largest interfering signal. Parallel to this work, Quek and et al. [28] developed a Poisson-based model of a heterogeneous network with arbitrary propagation effects (and single $\tau$ ) in order to study the probability of removing the $n$-th strongest signal and to decode the signal of interest after $n$ removals. They used two competing methods (based on Laplace transforms and truncated stable distributions) to derive approximations for the probability of decoding the $n$-th strongest signal given that the previous $n-1$ signals were decoded. The Laplace method was then used to derive an expression for the coverage probability (given $n$ signals are removed by interference cancellation), which agreed well with simulation results. In these works [25-28] the line of thought of using the order statistics of the propagation process arises, but it is then observed that such an approach appears quite intractable (an issue circumvented here by instead deriving and using the order statistics of the SINR process).

4) Factorial-moment expansion: In the theory of point processes factorial moment measures are an important set of tools that completely characterize a simple point process, hence they are used in stochastic geometry; see Daley and Vere-Jones [29, 30] or Stoyan et al. [31]. It has been shown that expectations of general functions of simple point processes can be written as (possibly infinite) expansions of the corresponding factorial moment measures, provided some convergence condition. This Taylor-like expansion theorem was first developed for unmarked point processes in one dimension by Błaszczyszyn [5], then generalized to higher dimensions by Błaszczyszyn et al. [6]. Kroese and Schmidt [32] specifically considered also independently marked point processes. It was recently applied in the setting of wireless networks to derive series expansions of the interference process induced by non-Poisson base station configurations [33]. Apart from these results, we are unaware of other work where factorial moment measure expansions have been used in a similar setting to that presented here.

To study the SINR process we define a related signal-to-total-interference-and-noise ratio (STINR) process which is more tractable. It turns out, this latter process is similar to (and, as recently observed in [34], in the case of a noiseless, an instance of) the well-studied Poisson-Dirichlet process. Pitman and Yor [35] surveyed in detail a generalized version of this process and derived various theoretical results. More recently, the factorial moment measures of this generalized version were derived

\footnotetext{
${ }^{3}$ It is tempting to suggest that some of these results [23] may form part of other work, based on the Poisson point process and a singular path-loss function, that holds under arbitrary propagation effects, but such a thorough and conclusive analysis is not part of this paper.
} 
by Handa [36], the work of which partly inspired us to derive the joint probability density of the order statistics of the SINR process, which we use to calculate the coverage probability under proposed signal combination and interference cancellation models.

\section{HETEROGENEOUS NETWORK MODEL AND QUANTITIES OF INTEREST}

We first present a cellular network model under which we define three related point processes on the positive half-line.

\section{A. Network and propagation model}

We consider the "typical user" approach where one assumes a typical user is located at the origin and examines what he perceives in the network. On $\mathbb{R}^{2}$, we model the base stations with a homogeneous or stationary Poisson point process $\Phi=\{X\}$ with density $\lambda$. Define the path-loss function as

$$
\ell(|x|)=(K|x|)^{\beta}
$$

with path-loss constant $K>0$ and path-loss exponent $\beta>2$. Given $\Phi$, let $\left\{\left(S_{X}, P_{X}\right)\right\}_{X \in \Phi}$ be a collection of independent (across $X$ ) and identically and arbitrarily distributed positive random vectors that form independent marks of $X$. Let $S_{X}$ represent the random propagation effects ${ }^{4}$ from the origin to $X$. For a signal emanating from a base station at $X$, let the $P_{X}$ represent the power of that signal. Let $(S, P)$ be equal in distribution to $\left\{\left(S_{X}, P_{X}\right)\right\}$ and note that $S$ and $P$ are not necessarily independent. We will refer to the above network (model) as a heterogeneous network (model).

\section{B. Propagation (loss) process}

We define the propagation (loss) process, considered as a point process on the positive half-line $\mathbb{R}^{+}$, as

$$
\Theta=\{Y\}:=\left\{\frac{\ell(|X|)}{P_{X} S_{X}}: X \in \Phi\right\},
$$

which is a Poisson process; for proof, see $[2,11]$ where $P$ is not random.

Lemma 1 (Propagation invariance). Assume that

$$
\mathbf{E}\left[(P S)^{\frac{2}{\beta}}\right]<\infty
$$

Then the propagation process $\{Y\}$ is an inhomogeneous Poisson point process with intensity measure $\Lambda([0, t))=$ at $t^{\frac{2}{\beta}}$, where the propagation constant is

$$
a:=\frac{\lambda \pi \mathbf{E}\left[(P S)^{\frac{2}{\beta}}\right]}{K^{2}} .
$$

Remark 2. In terms of propagation processes, this simple yet useful result allows one to represent propagation effects by setting the product $P S=1$, for example, and replacing $\lambda$ with $\lambda^{\prime}=\lambda \mathbf{E}\left[(P S)^{2 / \beta}\right]$; for more details see [7]. ${ }^{5}$

\section{SINR process}

We define the SINR process on the positive half-line $\mathbb{R}^{+}$for a typical user as

$$
\Psi=\{Z\}:=\{\operatorname{SINR}(X): X \in \Phi\},
$$

where

$$
\operatorname{SINR}(X):=\frac{Y^{-1}}{W+\gamma\left(I-Y^{-1}\right)},
$$

the constant $W \geq 0$ is the additive noise power, and

$$
I=\sum_{Y \in \Theta} Y^{-1}
$$

is the power received form the entire network (so that $I-Y^{-1}$ is the interference), and the constant $\gamma \in[0,1]$ is parameter that represents the "strength" of interference cancellation techniques. If we set $\gamma=0$, then the signal-to-noise ratio (SNR) process, $\operatorname{SNR}(X)=Y^{-1} / W$, is the inverse of the propagation (loss) process rescaled.

Remark 3. We consider the model that incorporates both the noise power $W$ and the "interference factor" $\gamma$. Note, however, that the SINR process with general $W>0, \gamma>0$ is equivalent to the SINR process rescaled by $\gamma^{-1}$ and evaluated for the noise power equal to $W \gamma^{-1}$ with the interference factor set to 1 . Thus $\gamma=1$ can be considered without loss of mathematical generality. We keep however this parameter to facilitate comparisons to some previous works.

\footnotetext{
${ }^{4} S$ may be written as a product of two random variables representing the fading and shadowing.

${ }^{5}$ The typical user approach coupled with the singular path-loss function (1) allows one to extend the model to $d$ dimensional space and also replace the constant density of base stations by an isotropic power-law function $r^{\alpha}$ with $-d<\alpha<\beta-d$. This generalization can be done by simply replacing $2 / \beta$ by $\alpha / \beta+d / \beta$ in the whole statement of Lemma 1 and $\pi / K^{2}$ by $\nu_{d} /\left((1+\alpha / d) K^{d}\right)$ in (4), where $\nu_{d}=\pi^{d / 2} / \Gamma(1+d / 2)$ is the volume of the unit-radius $d$-dimensional ball; cf e.g. [26, Lemma 1].
} 


\section{STINR process}

To study $\Psi$, it will be helpful to define the STINR process on $(0,1 / \gamma]$ as

$$
\Psi^{\prime}=\left\{Z^{\prime}\right\}:=\{\operatorname{STINR}(X): X \in \Phi\} .
$$

where

$$
\operatorname{STINR}(X):=\frac{Y^{-1}}{W+\gamma I},
$$

Note that the STINR is actually the signal-to-total-received-power-and-noise ratio. ${ }^{6}$ Naturally, working with $\Psi^{\prime}$ simplifies the algebra due to the common denominator in its definition and its bounded domain $(0,1 / \gamma)$. Information on $\Psi^{\prime}$ gives information on $\Psi$ by the relation

$$
Z=\frac{Z^{\prime}}{1-\gamma Z^{\prime}}
$$

or equivalently

$$
Z^{\prime}=\frac{Z}{1+\gamma Z}
$$

The STINR process reveals also some relations with some other, well studied mathematical and physical models as remarked in what follows.

Remark 4. Note that $\left\{Y^{-1}\right\}$ is an inhomogeneous Poisson process with intensity measure $(2 a / \beta) t^{-1-2 / \beta} d t$. By the construction (8) the STIR process $(W=0)$ with $\gamma=1$ is equal to, what is called in physics, a Poisson-Dirichlet process [38] with parameter $\theta=2 / \beta .{ }^{7}$ Much is known about the Poisson-Dirichlet process, including its factorial moment measures, but we cannot use these results in a straightforward manner in the case of STINR process (noisy model). However, some of our results (in Section V-A) are partly inspired by a recent approach to the Poisson-Dirichlet process presented in [36]. See [34] for more details on the relations between the two models and a few results, which can be directly derived using these relations.

\section{FACTORIAL MOMENT MEASURES OF THE SINR PROCESS}

We will derive the factorial moment measures of the STINR process $\left\{Z^{\prime}\right\}$ defined for $n \geq 1$ as

$$
M^{\prime(n)}\left(t_{1}^{\prime}, \ldots, t_{n}^{\prime}\right):=M^{\prime(n)}\left(\left(t_{1}^{\prime}, 1 / \gamma\right] \times \cdots \times\left(t_{n}^{\prime}, 1 / \gamma\right]\right)=\mathbf{E}\left(\sum_{\substack{\left(Z_{1}^{\prime}, \ldots, Z_{n}^{\prime}\right) \in\left(\Psi^{\prime}\right) \times n \\ \text { distinct }}} \prod_{j=1}^{n} \mathbb{1}\left(Z_{j}^{\prime}>t_{j}^{\prime}\right)\right),
$$

where $\mathbb{1}$ denotes the indicator function. The factorial measures of $\left\{Z^{\prime}\right\}$, which in turn give those of $\{Z\}$, not only completely characterize these processes, but allows one to express in a natural manner the probabilities of various events related to SINR coverage, as we shall see in this paper.

Before deriving $M^{\prime(n)}$, we introduce two useful integrals, the first of which arose in the $k$-coverage problem [18] while the second is a generalization of another integral in the same work. For $x \geq 0$ define

$$
\mathcal{I}_{n, \beta}(x)=\frac{2^{n} \int_{0}^{\infty} u^{2 n-1} e^{-u^{2}-u^{\beta} x \Gamma(1-2 / \beta)^{-\beta / 2}} d u}{\beta^{n-1}\left(C^{\prime}(\beta)\right)^{n}(n-1) !}
$$

where

$$
C^{\prime}(\beta)=\frac{2 \pi}{\beta \sin (2 \pi / \beta)}=\Gamma(1-2 / \beta) \Gamma(1+2 / \beta) .
$$

Note that

$$
\mathcal{I}_{n, \beta}(0)=\frac{2^{n-1}}{\beta^{n-1}\left(C^{\prime}(\beta)\right)^{n}}
$$

For all $x_{i} \geq 0$ define

$$
\mathcal{J}_{n, \beta}\left(x_{1}, \ldots, x_{n}\right)=\frac{\left(1+\sum_{j=1}^{n} x_{j}\right)}{n} \int_{[0,1]^{n-1}} \frac{\prod_{i=1}^{n-1} v_{i}^{i(2 / \beta+1)-1}\left(1-v_{i}\right)^{2 / \beta}}{\prod_{i=1}^{n}\left(x_{i}+\eta_{i}\right)} d v_{1} \ldots d v_{n-1},
$$

\footnotetext{
${ }^{6}$ Total interference means the total power received from all transmitters, including the useful signal; cf [37, Notes, page 204].

${ }^{7}$ It appears as the thermodynamic (large system) limit in the low temperature regime of Derrida's random energy model and a key component of the so-called Ruelle probability cascades, which are used to represent the thermodynamic limit of the Sherrington-Kirkpatrick model for spin glasses. It should not be confused with the (perhaps) better known Poisson-Dirichlet process of Kingman [39] for which $\left\{Y^{-1}\right\}$ should be Poisson process with intensity (measure) $\alpha t^{-1} e^{-t} d t$ with some constant $\alpha>0$. In fact both processes are special cases of two-parameter Poisson-Dirichlet process extensively studied in [35].
} 
where

$$
\left\{\begin{aligned}
\eta_{1} & =v_{1} v_{2} \ldots v_{n-1} \\
\eta_{2} & =\left(1-v_{1}\right) v_{2} \ldots v_{n-1} \\
\eta_{3} & =\left(1-v_{2}\right) v_{3} \ldots v_{n-1} \\
& \cdots \\
\eta_{n} & =1-v_{n-1} .
\end{aligned}\right.
$$

Remark 5. It is not straightforward to observe (but follows from Theorem 7) that $\mathcal{J}_{n, \beta}\left(x_{1}, \ldots, x_{n}\right)$ is invariant under any variable permutation. It has been defined to be analogous to the single-variable version $\mathcal{J}_{n, \beta}(x)$ found in [18], with $\mathcal{J}_{n, \beta}(x, \ldots, x)=\mathcal{J}_{n, \beta}(x)$. For more remarks on $\mathcal{J}_{n, \beta}\left(x_{1}, \ldots, x_{n}\right)$, including its representation as a functional of $n-1$ independent beta random variables see Appendix A.

We now observe that the factorial moment measure is zero outside a simplex defined by $t_{i}^{\prime}$ values.

Lemma 6. For $t_{i}^{\prime} \in(0,1 / \gamma]$, the factorial moment measure of the STINR process (8) satisfies

$$
M^{\prime(n)}\left(t_{1}^{\prime}, \ldots, t_{n}^{\prime}\right)=0
$$

when

$$
\gamma \sum_{i=1}^{n} t_{i}^{\prime} \geq 1
$$

Proof. This is a direct consequence of a well-known result [40, Proposition 6.2], which states that if the intersection of SINR cells [40, Definition 5.1] is not empty, then $\sum_{i=1}^{n} t_{i}^{\prime} \leq 1 / \gamma$.

We define

$$
\hat{t}_{i}=\hat{t}_{i}\left(t_{1}^{\prime}, \ldots, t_{n}^{\prime}\right):=\frac{\gamma t_{i}^{\prime}}{1-\gamma \sum_{j=1}^{n} t_{j}^{\prime}}
$$

and present the main result, which characterizes the STINR process (8).

Theorem 7 (Factorial moment measure of $\left.\Psi^{\prime}\right)$. Assume that moment condition (3) holds. Then for $t_{i}^{\prime} \in(0,1 / \gamma]$, the factorial moment measure of order $n \geq 1$ of the STINR process (8) satisfies

$$
M^{\prime(n)}\left(t_{1}^{\prime}, \ldots t_{n}^{\prime}\right)=n !\left(\prod_{i=1}^{n} \hat{t}_{i}^{-2 / \beta}\right) \mathcal{I}_{n, \beta}\left((W / \gamma) a^{-\beta / 2}\right) \mathcal{J}_{n, \beta}\left(\hat{t}_{1}, \ldots, \hat{t}_{n}\right)
$$

when

$$
\gamma \sum_{i=1}^{n} t_{n}^{\prime}<1
$$

and

$$
M^{\prime(n)}\left(t_{1}^{\prime}, \ldots t_{n}^{\prime}\right)=0
$$

otherwise.

Proof. Expression (23) is due to Lemma 6. For expression (21), the proof is included in Appendix B. It follows in a similar fashion to that of the main theorem in [18] .

We immediately obtain the moment measure of the SINR process, which is defined by

$$
M^{(n)}\left(t_{1}, \ldots t_{n},\right):=M^{(n)}\left(\left(t_{1}, \infty\right] \times \cdots \times\left(t_{n}, \infty\right]\right)=\mathbf{E}\left(\sum_{\substack{\left.Z_{1}, \ldots, Z_{n}\right) \in(\Psi) \times n \\ \text { distinct }}} \prod_{j=1}^{n} \mathbb{1}\left(Z_{j}>t_{j}\right)\right) .
$$

Corollary 8 (Factorial moment measure of $\Psi$ ). Assume the propagation moment condition (3). Then for $t_{i} \in(0, \infty)$, the SINR process (5) has the moment measure

$$
M^{(n)}\left(t_{1}, \ldots t_{n},\right)=M^{\prime(n)}\left(t_{1}^{\prime}, \ldots, t_{n}^{\prime}\right)
$$

where

$$
t_{i}^{\prime}=\frac{t_{i}}{1+\gamma t_{i}}
$$

Proof. The result follows from the relationship between $\Psi_{i}$ and $\Psi_{i}^{\prime}$ captured in expressions (10) and (24). 
Remark 9. [Noise factorization] Denote by $M_{W=0, \gamma=1}^{\prime(n)}$ and $M_{W=0, \gamma=1}^{(n)}$ respectively the factorial moment measures of the STIR and SIR processes with $W=0$ and $\gamma=1$. By (21), Remark 3 and Corollary 8 we have immediately the following factorization of the noise in the factorial moment measures with arbitrary $W$ and $\gamma$

$$
\begin{aligned}
M^{\prime(n)}\left(t_{1}^{\prime}, \ldots, t_{n}^{\prime}\right) & =\overline{\mathcal{I}}_{n, \beta}\left((W / \gamma) a^{-\beta / 2}\right) M_{W=0, \gamma=1}^{\prime(n)}\left(\gamma t_{1}^{\prime}, \ldots, \gamma t_{n}^{\prime}\right), \\
M^{(n)}\left(t_{1}, \ldots, t_{n}\right) & =\overline{\mathcal{I}}_{n, \beta}\left((W / \gamma) a^{-\beta / 2}\right) M_{W=0, \gamma=1}^{(n)}\left(\gamma t_{1}, \ldots, \gamma t_{n}\right),
\end{aligned}
$$

where

$$
\overline{\mathcal{I}}_{n, \beta}(x)=\frac{\mathcal{I}_{n, \beta}(x)}{\mathcal{I}_{n, \beta}(0)} .
$$

This ability to factor out the noise effect in the factorial moment measures is convenient and is reminiscent of factoring out the noise term in the distribution of the SINR under Rayleigh fading, an assumption that is not required, however, in our present setting. In particular it allows one to express the densities of the factorial moment measures $M^{\prime(n)}$ of the STINR process as follows, using the corresponding densities of the Poisson-Dirichlet process from [36, Theorem 2.1]; cf Remark 4. For $n \geq 0$ denote

$$
c_{n, \alpha, \theta}=\prod_{i=1}^{n} \frac{\Gamma(\theta+1+(i-1) \alpha)}{\Gamma(1-\alpha) \Gamma(\theta+i \alpha)},
$$

in particular $c_{n, 2 / \beta, 0}=(2 / \beta)^{n-1} \Gamma(n) /\left(\Gamma(2 n / \beta) \Gamma(1-2 / \beta)^{n}\right)$.

Corollary 10. For the STINR process $\Psi^{\prime}(W \geq 0)$, the $n$th factorial moment density is given by

$$
\begin{aligned}
& \mu^{\prime(n)}\left(t_{1}^{\prime}, \ldots t_{n}^{\prime}\right):=(-1)^{n} \frac{\partial^{n} M^{\prime(n)}\left(t_{1}^{\prime}, \ldots t_{n}^{\prime}\right)}{\partial t_{1}^{\prime} \ldots \partial t_{n}^{\prime}} \\
= & c_{n, 2 / \beta, 0} \overline{\mathcal{I}}_{n, \beta}\left((W / \gamma) a^{-\beta / 2}\right) \gamma^{n}\left(\prod_{i=1}^{n}\left(\gamma t_{i}^{\prime}\right)^{-(2 / \beta+1)}\right)\left(1-\sum_{j=1}^{n}\left(\gamma t_{j}^{\prime}\right)\right)^{2 n / \beta-1}
\end{aligned}
$$

for $\left(t_{1}^{\prime}, \ldots, t_{n}^{\prime}\right)$ satisfying (22) and 0 otherwise.

We are unaware of anybody showing the equivalence of Propositions 7 and Corollary 10, either by differentiating the measure (21) or integrating the density (30).

\section{COVERAGE PROBABILITIES IN HETEROGENEOUS NETWORKS WITH VARYING SINR THRESHOLDS}

In this section we will present a result in which the factorial moments of the SINR process naturally arise, thus illustrating an intuitive and convincing reason for their introduction before applying them in a more general setting. We will see that the $k$-coverage probability framework introduced in [18] holds for very general settings. In this regard, we will define marked versions of the previously introduced point processes with the inclusion of SINR threshold values. Informally, we will refer to a network (model) in which each value of $S, P$ and the SINR threshold is random and depends on each base station as a heterogeneous network (model) with varying SINR thresholds.

\section{A. Heterogeneous network model with varying SINR thresholds}

Given $\Phi=\{X\}$, let $\left\{T_{X}\right\}_{X \in \Phi}$ be a collection of positive random variables that each represent the SINR threshold of a base station. For each $X \in \Phi$, the positive random vector $\left(S_{X}, P_{X}, T_{X}\right)$ forms its independent mark. Our network model is now described by the independently marked point process

$$
\widetilde{\Phi}:=\left\{\left(X,\left(S_{X}, P_{X}, T_{X}\right)\right)\right\}
$$

Note that the coordinates of $\left(S_{X}, P_{X}, T_{X}\right)$ are not necessarily independent to each other. The marked process $\widetilde{\Phi}$ induces the independently marked propagation process

$$
\widetilde{\Theta}:=\{(Y, T)\}=\left\{\left(\frac{\ell(X)}{P_{X} S_{X}}, T_{X}\right)\right\} .
$$

Effectively, what the typical user 'experiences' in a heterogeneous network is captured by the independently marked point process $\widetilde{\Theta}$. This process admits a propagation invariance result [7], analogous to Lemma 1, which was originally given with random $\beta$, but is now presented with constant $\beta$ (see Appendix $\mathrm{E}$ for proof).

Lemma 11 (Marked propagation invariance). Assume that

$$
\mathbf{E}\left[(P S)^{2 / \beta}\right]<\infty .
$$


Then the propagation process $\widetilde{\Theta}$ is an independently marked inhomogeneous Poisson point process on $\mathbb{R}_{+}$with intensity measure

$$
\Lambda(s, t):=\mathbf{E}\left[\sum_{(Y, T) \in \widetilde{\Theta}} \mathbb{1}(Y \leq s, T \leq t)\right]=\frac{\lambda \pi s^{2 / \beta}}{K^{2}} \mathbf{E}\left[(P S)^{2 / \beta} \mathbb{1}(T \leq t)\right] .
$$

If two network models induce the same marked propagation processes $\widetilde{\Theta}$, then we say they are stochastically equivalent. Furthermore, two equivalent networks also induce the same independently marked SINR process

$$
\widetilde{\Psi}:=\{(Z, T)\} \text {. }
$$

where $Z=\operatorname{SINR}(X)$ as given by expression (6).

Lemma 11 allows one to construct an equivalent network with some of the previously random marks set to constants. There is a subtlety in this ability to 'push' randomness away from certain marks onto others that serves as a useful technique in proofs, which we leverage in the next result.

Corollary 12. For the network $\widetilde{\Phi}$, there is a stochastically equivalent network

$$
\widetilde{\Phi}^{*}=\left\{\left(X^{*},\left(S_{X^{*}}=1, P_{X^{*}}=1, T_{X^{*}}\right)\right)\right\},
$$

with density

$$
\lambda^{*}:=\lambda \mathbf{E}\left[(P S)^{2 / \beta}\right]=a K^{2} / \pi,
$$

where given $\left\{\left(X^{*}\right)\right\}$, the marks $T_{X^{*}}$ are independent (across $\left.X^{*}\right)$, and with a distribution given by

$$
F_{T^{*}}:=\mathbf{P}\left(T^{*} \leq t\right)=\frac{\mathbf{E}\left[(P S)^{2 / \beta} \mathbb{1}(T \leq t)\right]}{\mathbf{E}\left[(P S)^{2 / \beta}\right]} .
$$

Consequently, $\widetilde{\Phi}^{*}$ induces the marked SINR process

$$
\widetilde{\Psi}^{*}:=\left\{\left(Z^{*}, T^{*}\right)\right\},
$$

which is equal in distribution to $\widetilde{\Psi}$, hence the factorial moment measures of $\left\{Z^{*}\right\}$ are equal to these of $\{Z\}$, namely $M^{(n)}$ with propagation constant $a=\pi \lambda^{*} / K^{2}$. The same is true for the STINR process induced by $\widetilde{\Phi}^{*}$.

Proof. Set the marks to $S=1$ and $P=1$, substitute $F_{T^{*}}$, and verify that the resulting propagations process forms a marked Poisson process with intensity measure $\Lambda(s, t)$ given by (33).

\section{B. Coverage number and symmetric sums}

We now introduce some quantities of interest that first appeared in the single-tier case [18], but hold under the more general setting of heterogeneous networks. We consider the coverage number of the typical user, which is defined as the number of base stations to which the typical user can connect, namely

$$
\mathcal{N}:=\sum_{(Z, T) \in \widetilde{\Psi}} \mathbb{1}[Z>T] .
$$

The probability of the typical user being covered by at least $k$ base stations, or the $k$-coverage probability, is

$$
\mathcal{P}^{(k)}:=\mathbf{P}\{\mathcal{N} \geq k\}
$$

In particular, the coverage probability of the typical user is $\mathcal{P}:=\mathcal{P}^{(1)}$. In the spirit of the previous $k$-coverage result [18], we introduce the notion of symmetric sums. For $n \geq 1$, define the $n$th symmetric sum

$$
\mathcal{S}_{n}:=\mathbf{E}\left[\sum_{\substack{\left(Z_{i}, T_{i}\right) \in(\widetilde{\Psi}) \times n \\ \text { distinct }}} \mathbb{1}\left(Z_{i}>T_{i}, i=1, \ldots, n\right)\right]
$$

We set $\mathcal{S}_{0}:=1$. $\mathcal{S}_{n}$ represents the expected number of ways of choosing $n$ different base stations $X_{1}, \ldots, X_{m}$ each received by the user at the origin with the SINR larger than it specific value $T_{X_{i}}$. We can call it the expected n-coverage number.

The following $\mathcal{S}_{n}$-based identities are akin to the famous inclusion-exclusion principle (for example, see [41, IV.5 and IV.3] for (42) and (43), respectively) and stem from the Schuette-Nesbitt formula (which we recall for completeness in Appendix F, see also [42]). 
Lemma 13. We have for $k \geq 1$

$$
\begin{aligned}
\mathcal{P}^{(k)} & =\sum_{n=k}^{\infty}(-1)^{n-k}\left(\begin{array}{l}
n-1 \\
k-1
\end{array}\right) \mathcal{S}_{n}, \\
\mathbf{P}\{\mathcal{N}=k\} & =\sum_{n=k}^{\infty}(-1)^{n-k}\left(\begin{array}{l}
n \\
k
\end{array}\right) \mathcal{S}_{n}, \\
\mathbf{E}\left[z^{\mathcal{N}}\right] & =\sum_{n=0}^{\infty}(z-1)^{n} \mathcal{S}_{n}, \quad z \in[0,1], \\
\mathbf{E}[\mathcal{N}] & =\mathcal{S}_{1} .
\end{aligned}
$$

We will see, via Lemma 15, that the above (apparently infinite) summations reduce to finite sums (owing to $\mathcal{S}_{n}=0$ for large enough $n$ ) under very reasonable model conditions.

Proposition 14. For the network $\widetilde{\Phi}$, under the assumptions of Theorem 7 , the $n$th symmetric sum is

$$
\mathcal{S}_{n}=\frac{1}{n !} \int_{\left(\mathbb{R}_{+}\right)^{n}}\left(\prod_{i=1}^{n} F_{T^{*}}\left(t_{i}\right)\right) M^{(n)}\left(d t_{1}, \ldots, d t_{n}\right),
$$

which is equivalent to

$$
\mathcal{S}_{n}=\frac{1}{n !} \int_{\left(\mathbb{R}_{+}\right)^{n}} M^{(n)}\left(t_{1}, \ldots, t_{n}\right) \prod_{i=1}^{n} F_{T^{*}}\left(d t_{i}\right),
$$

where $M^{(n)}$ and $F_{T^{*}}$ are given by (25) and (37) respectively.

Proof. Corollary 12 implies

$$
\mathcal{S}_{n}:=\mathbf{E}\left[\sum_{\substack{\left(Z_{i}^{*}, T_{i}^{*}\right) \in\left(\widetilde{\Psi}^{*}\right) \times n \\ \text { distinct }}} \mathbb{1}\left(Z_{i}^{*}>T_{i}^{*}, i=1, \ldots, n\right)\right] .
$$

Denote by $\Psi^{*}=\left\{Z^{*}\right\}$ the process of points of $\widetilde{\Psi}^{*}$. With this notation, using the fact that $\widetilde{\Psi}^{*}$ is independently marked, we have

$$
\begin{aligned}
\mathcal{S}_{n} & =\mathbf{E}\left[\sum_{\substack{\left(Z_{i}^{*}\right) \in\left(\Psi^{*}\right) \times n \\
\text { distinct }}} \mathbf{P}\left(Z_{i}^{*}>T_{i}^{*}, i=1, \ldots, n \mid \Psi^{*}\right)\right] \\
& =\mathbf{E}\left[\sum_{\substack{\left(Z_{i}^{*}\right) \in\left(\Psi^{*}\right) \times n \\
\text { distinct }}} \prod_{i=1}^{n} F_{T^{*}}\left(Z_{i}^{*}\right)\right] \\
& =\frac{1}{n !} \int_{\left(\mathbb{R}_{+}\right)^{n}}\left(\prod_{i=1}^{n} F_{T^{*}}\left(z_{i}\right)\right) M^{(n)}\left(d z_{1}, \ldots, d z_{n}\right),
\end{aligned}
$$

where the last line follows from Campbell's theorem for marked point processes and the definition of factorial moment measures [31, Chapter 4]. For equation (47), by Lemma 6 and Corollary 8,

$$
\lim _{t_{i} \rightarrow \infty} M^{(n)}\left(t_{1}, \ldots, t_{n}\right)=0, \quad i \in[1, n] .
$$

Note that $M^{(n)}\left(t_{1}, \ldots, t_{n}\right)$ is a decreasing function in $t_{i}$, and

$$
\lim _{t_{1} \rightarrow 0} F_{T^{*}}\left(t_{i}\right)=0 \text {. }
$$

Then apply integration by parts

$$
\int_{\left(\mathbb{R}_{+}\right)^{n}} M^{(n)}\left(t_{1}, \ldots, t_{n}\right) \prod_{i=1}^{n} F_{T^{*}}\left(d t_{i}\right)=\int_{\left(\mathbb{R}_{+}\right)^{n}} M^{(n)}\left(d t_{1}, \ldots, t_{n}\right) F_{T^{*}}\left(t_{1}\right) \prod_{i=2}^{n} F_{T^{*}}\left(d t_{i}\right),
$$

and repeat until proof is completed.

The next result shows that the expressions involving infinite sums of $\mathcal{S}_{n}$ reduce to finite sums. For real $x$ denote by $\lceil x\rceil$ the ceiling of $x$ (the smallest integer not less than $x$ ).

Lemma 15. Assume there exists $t_{\text {min }}>0$ such that $\mathbf{P}\left(T \geq t_{\text {min }}\right)=1$. Then $\mathcal{S}_{n}=0$ for $n \geq 1 /\left(\gamma t_{\text {min }}\right)+1$, which implies that one can replace $\infty$ by $\left\lceil 1 /\left(\gamma t_{\min }\right)\right\rceil$ in the sums in expressions given in Lemma 13, namely equations (42)-(44)

Proof. If $T \geq t_{\min }$ almost surely, then the same holds true for $T^{*}$. Consequently by (47) and Lemma $6 \mathcal{S}_{n}=0$ when $n \gamma t_{\min } /\left(1+\gamma t_{\min }\right) \geq 1$. The largest $n$ for which the opposite, strict inequality holds is $\left\lceil 1 /\left(\gamma t_{\min }\right)\right\rceil$. 


\section{Multi-tier network example}

We illustrate our $k$-coverage framework by examining an increasingly popular multi-tier cellular network model. Informally, we refer to a heterogeneous network as a multi-tier network if the marks $T$ are set to constants that depend only on the tier to which the base station belongs.

On $\mathbb{R}^{2}$ we represent $m$ tiers of base stations with $m$ independent homogeneous Poisson point processes $\left\{\Phi_{j}\right\}$ with densities $\left\{\lambda_{j}\right\}$. More precisely, consider the independently marked point process

$$
\widetilde{\Phi}_{j}:=\left\{\left(X_{j},\left(S_{X_{j}}, P_{X_{j}}, \tau_{j}\right)\right)\right\},
$$

where the SINR threshold mark has been set to a non-random $\tau_{j}$, and where the distribution of the random vector $\left(P_{j}, S_{j}\right)$ is equal to that of $\left(S_{X_{j}}, P_{X_{j}}\right)$, such that the base station power and propagation effects may depend on the tier, and

$$
\mathbf{E}\left[\left(P_{j} S_{j}\right)^{2 / \beta}\right]<\infty, \quad j=1, \ldots, m .
$$

Assume without loss of generality that $\tau_{j} \neq \tau_{k}$ for $j \neq k$. Let $K$ and $\beta$ again be the path-loss parameters for the entire network, and denote

$$
\lambda_{j}^{*}=\lambda_{j} \mathbf{E}\left[\left(P_{j} S_{j}\right)^{2 / \beta}\right] .
$$

We call the superposition $\widetilde{\Phi}:=\cup_{j=1}^{m} \widetilde{\Phi}_{j}$ the $m$-tier network model.

Corollary 16. An equivalent (to $\widetilde{\Phi}$ ) single-tier network $\widetilde{\Phi}^{*}$ exists with the path-loss exponent $\beta$, path-loss constant $K=1$, assumes $P^{*} \equiv 1, S^{*} \equiv 1$, and a base station density given by

$$
\lambda^{*}=\sum_{j=1}^{m} \lambda_{j}^{*},
$$

and marks $T^{*}$ with distribution

$$
\mathbf{P}\left(T^{*}=\tau_{j}\right)=\frac{\lambda_{j}^{*}}{\lambda^{*}}, \quad j=1, \ldots, m .
$$

Proof. For each $j$ tier $\widetilde{\Phi}_{j}$, there is an equivalent tier $\widetilde{\Phi}_{j}^{*}$ with $P_{j}=1, S_{j}=1$, and density $\lambda_{i}^{*}$. Using the superposition theorem [39], the result follows from Corollary 12.

Remark 17. Note that a "randomly" selected base station of $\Phi$ belongs to the $j$ th tier with probability $\lambda_{j} / \lambda$, where $\lambda=$ $\sum_{j=1}^{m} \lambda_{j}$, while a "randomly" selected signal from the propagation process $\left\{Z^{*}\right\}$ originates from a base station in the $j$ th tier with probability $\lambda_{j}^{*} / \lambda^{*}$.

The following result gives an explicit $\mathcal{S}_{n}$ expression for the multi-tier model with arbitrary propagation effects in the domain of all $t_{i}>0$, and thus, in conjunction with Lemma 13, allows one to calculate the $k$-coverage probabilities, which generalizes previous results $[14,15,18]$.

Corollary 18. Assume propagation moment condition (3) for all $m$ tiers of a mult-tier network. Then

$$
\mathcal{S}_{n}=\frac{1}{n !}\left[\sum_{\substack{j \in\{1, \ldots, n\} \\ i_{j} \in\{1, \ldots, m\}}} M^{(n)}\left(\tau_{i_{1}}, \ldots, \tau_{i_{n}}\right) \prod_{j=1}^{n} \lambda_{i_{j}}^{*} / \lambda^{*}\right] .
$$

Proof. This stems directly from Corollary 16 amd expression (47).

\section{Single-tier network example}

For the special case of a single-tier stationary Poisson network model $\Phi=\{X\}$ with density $\lambda$ and a (deterministic) SINR threshold $\tau$ the $k$-coverage probability expressions derived in [18] follow. The second statement (case with $\gamma \tau \geq 1$ ) is a special case of [14, Theorem 1].

Corollary 19. Assume the moment condition $\mathbf{E}\left[(P S)^{2 / \beta}\right]<\infty$ for a single-tier network. Then

$$
\mathcal{S}_{n}=\mathcal{S}_{n}(\tau)=\tau_{n}^{-2 n / \beta} \mathcal{I}_{n, \beta}\left((W / \gamma) a^{-\beta / 2}\right) \mathcal{J}_{n, \beta}\left(\tau_{n}\right),
$$

for $0<\gamma \tau<1 /(n-1)$ and $\mathcal{S}_{n}=0$ otherwise, where a is given by (4) and $\tau_{n}$ is given by

$$
\tau_{n}:=\tau_{n}(\tau)=\frac{\gamma \tau}{1-(n-1) \gamma \tau},
$$


Moreover, the k-coverage probability is

$$
\mathcal{P}^{(k)}=\mathcal{P}^{(k)}(\tau)=\sum_{n=k}^{\lceil 1 /(\gamma \tau)\rceil}(-1)^{n-k}\left(\begin{array}{c}
n-1 \\
k-1
\end{array}\right) \tau_{n}^{-2 n / \beta} \mathcal{I}_{n, \beta}\left((W / \gamma) a^{-\beta / 2}\right) \mathcal{J}_{n, \beta}\left(\tau_{n}\right),
$$

For $\gamma \tau \geq 1$ we have $\lceil 1 /(\gamma \tau)\rceil=1$ and

$$
\mathcal{P}^{(1)}(\tau)=\frac{2(\gamma \tau)^{-2 / \beta}}{\Gamma\left(1+\frac{2}{\beta}\right)} \int_{0}^{\infty} u e^{-u^{2} \Gamma(1-2 / \beta)-(W / \gamma) a^{-\beta / 2} u^{\beta}} d u
$$

Remark 20. If we set $W=0$ (to consider an "interference limited network"), then $\{Z\}$ becomes the SIR process, which is clearly scale-invariant due to the definition of the regular and STIR process in terms of the propagation process (also evident by the factorial moment measures being independent of $\lambda$ ). Under the stationary single-tier Poisson model with $\gamma=1$, the inverse of the largest SIR value, known as the interference factor $f$, has been studied [2] by deriving its Laplace transform

$$
\mathcal{L}_{f}(\xi):=\mathbf{E}\left[e^{-\xi f}\right]=\frac{1}{\varphi_{\beta}(\xi)}
$$

where

$$
\varphi_{\beta}(\xi):=e^{-\xi}+\xi^{2 / \beta} \gamma(1-2 / \beta, \xi)
$$

and $\gamma(z, \xi)=\int_{0}^{\xi} t^{z-1} e^{-t} d t$ is the lower incomplete gamma function. The distribution of $f$ is directly related to $\mathcal{P}$ (in the interference limited network) by $\mathbf{P}(f \leq s)=\mathcal{P}(1 / s)$, which gives the Laplace transform relation

$$
\xi \int_{0}^{\infty} \mathcal{P}(1 / s) e^{-\xi s} d s=\mathcal{L}_{f}(\xi)=\frac{1}{\varphi_{\beta}(\xi)}
$$

\section{FINITE-DIMENSIONAL DISTRIBUTIONS OF THE SINR PROCESS}

In this section we show how the factorial moment measures of the SINR process can be used to derive joint densities of the $k$ strongest values of the SINR process. This allows one to express, for example, coverage probabilities under interference cancellation and/or base station cooperation.

\section{A. Joint densities of order statistics}

Denote the order statistics of the STINR process $\Psi^{\prime}=\left\{Z^{\prime}\right\}$ by

$$
Z_{(1)}^{\prime}>Z_{(2)}^{\prime}>Z_{(3)}^{\prime} \cdots,
$$

such that $Z_{(1)}^{\prime}$ is the largest STINR value in $\Psi^{\prime}$. In complete analogy one can consider order statistics $Z_{(1)}>Z_{(2)}>Z_{(3)} \ldots$ of the SINR process $\Psi=\{Z\}$. However, as we shall see, $\Psi^{\prime}$ is a more apt tool when studying interference cancellation and base station cooperation.

Remark 21. By its definition $(40), \mathcal{P}^{(k)}(\tau)$ evaluated in Corollary 19, as a function of $\tau$, is the tail-distribution of the $k$ th order statistics of the SINR process, $\mathcal{P}^{(k)}(\tau)=\mathbf{P}\left\{Z_{(k)}>\tau\right\}$. By the fact that the mappings (10) and (11) are monotonic, increasing, $\mathcal{P}^{(k)}\left(\tau^{\prime} /\left(1-\gamma \tau^{\prime}\right)\right)=\mathbf{P}\left\{Z_{(k)}^{\prime}>\tau^{\prime}\right\}$. Moreover, given that the functions $x / A$ and $x /(A-x)=A /(A-x)-1$ are increasing in $x, Z_{(i)}$ (or $Z_{(i)}^{\prime}$ ) represent the value of the SINR (or STINR) experienced by the typical user with respect to the base station offering the $k$ th smallest propagation loss $Y^{(k)}$, where $Y^{(1)}<Y^{(2)}<\ldots$ is the process of order statistics of $\{Y\}$.

We will express now the joint probability density $f_{(k)}^{\prime}\left(z_{1}^{\prime}, \ldots, z_{k}^{\prime}\right)$ of the vector $\left(Z_{(1)}^{\prime}, \ldots, Z_{(k)}^{\prime}\right)$ of $k(k \geq 1)$ largest order statistics of the STINR process.

For $i \geq 1$, write

$$
\mu_{k}^{\prime(k+i)}\left(z_{1}^{\prime}, \ldots, z_{k}^{\prime}\right)=\int_{z_{k}^{\prime}}^{1 / \gamma} \ldots \int_{z_{k}^{\prime}}^{1 / \gamma} \mu^{\prime(k+i)}\left(z_{1}^{\prime}, \ldots, z_{k}^{\prime}, \zeta_{1}^{\prime}, \ldots, \zeta_{i}^{\prime}\right) d \zeta_{i}^{\prime} \ldots d \zeta_{1}^{\prime},
$$

where $\mu^{\prime(n)}\left(t_{1}^{\prime}, \ldots, t_{n}^{\prime}\right)=(-1)^{n} \frac{\partial^{n} M^{\prime(n)}}{\partial t_{1}^{\prime} \ldots \partial t_{n}^{\prime}}\left(t_{1}^{\prime}, \ldots, t_{n}^{\prime}\right)$ is the density of the factorial moment measure $M^{\prime(n)}$ of the STINR process given in Corollary 10. It is easy to see that (60) can be evaluated more directly differentiating $k$ times $M^{\prime(n)}$

$$
\mu_{k}^{\prime(k+i)}\left(z_{1}^{\prime}, \ldots, z_{k}^{\prime}\right)=(-1)^{k} \frac{\partial^{k} M^{\prime(k+i)}\left(t_{1}^{\prime}, \ldots, t_{k}^{\prime}, z_{k}^{\prime}, \ldots, z_{k}^{\prime}\right)}{\partial t_{1}^{\prime} \ldots \partial t_{k}^{\prime}}\left(t_{1}^{\prime}=z_{1}^{\prime}, \ldots, t_{k}^{\prime}=z_{k}^{\prime}\right) .
$$

For convenience we define also $\mu_{k}^{\prime(k+i)}$ for $i=0$ as the density of $M^{\prime(k)} ; \mu_{k}^{\prime(k)}:=\mu^{\prime(k)}$. We will call $\mu_{k}^{\prime(n)}(k \leq n)$ "partial densities" of $M^{\prime(n)}$. In Appendix C and D we will show how these partial densities can be explicitly calculated.

The equivalent notations apply to the SINR process $\Psi=\{Z\}$, which we skip without loss of completeness. 
Proposition 22. Under the assumptions of Theorem 7, the joint probability density of the vector of $k$ strongest values of the STINR process $\left(Z_{(1)}^{\prime}, \ldots, Z_{(k)}^{\prime}\right)$ is equal to

$$
f_{(k)}^{\prime}\left(z_{1}^{\prime}, \ldots, z_{k}^{\prime}\right)=\sum_{i=0}^{i_{\max }} \frac{(-1)^{i}}{i !} \mu_{k}^{\prime(k+i)}\left(z_{1}^{\prime}, \ldots, z_{k}^{\prime}\right),
$$

for $z_{1}^{\prime}>z_{2}^{\prime}>\cdots>z_{k}^{\prime}$ and $f_{(k)}^{\prime}\left(z_{1}^{\prime}, \ldots, z_{k}^{\prime}\right)=0$ otherwise, where the upper summation limit is bounded by

$$
i_{\max }<\frac{1}{\gamma z_{k}^{\prime}}-k
$$

Proof. The result is effectively that of Handa [36, Lemma 5.3] who details how it follows from a relationship between finite point processes and their Janossy measures (see [29, Section 5.4]). The original result has the infinite series in (62). However, by Lemma $6 M^{\prime(i+k)}\left(t_{1}^{\prime}, \ldots, t_{k}^{\prime}, z_{k}^{\prime}, \ldots, z_{k}^{\prime}\right)=0$ when $t_{1}^{\prime}+\ldots+t_{k}^{\prime}+i z_{k} \geq 1 / \gamma$ and consequently, by $(61), \mu_{k}^{(k+i)}\left(z_{1}^{\prime}, \ldots, z_{k}^{\prime}\right)=0$ whenever $z_{1}^{\prime}+\ldots+z_{k-1}^{\prime}+(i+1) z_{k}^{\prime} \geq 1 / \gamma$. Hence, a necessary condition for $\mu_{k}^{(k+i)}\left(z_{1}^{\prime}, \ldots, z_{k}^{\prime}\right)>0$ is $z_{1}^{\prime}+\ldots(i+1) z_{k}^{\prime}<1 / \gamma$ which, since $z_{1}^{\prime}>\cdots>z_{k}^{\prime}$, implies $(k+i) z_{k}^{\prime}<1 / \gamma$. This proves (63). The original result of Handa has also a convergence condition

$$
\sum_{n=0}^{\infty} \frac{M^{\prime(n)}(c, \ldots, c)}{n !}(1+\varepsilon)^{n}<\infty
$$

for each $c \in(0,1 / \gamma)$ and some $\varepsilon=\varepsilon(c)$, which is trivially satisfied in our case by the previous observation regarding $i_{\max }$.

The bound on $i_{\max }$ given in (63) is only an upper bound of the maximum index of non-zero terms of the expansion of $f_{(k)}^{\prime}\left(t_{1}^{\prime}, \ldots, t_{k}^{\prime}\right)$ in terms of the partial densities of the factorial moment measures. For some particular domains of $\left(t_{1}^{\prime}, \ldots, t_{k}^{\prime}\right)$, a smaller value of $i_{\max }$ can be given; cf the proof of Proposition 25 in the next section.

Using Proposition 22 and Corollary 10 we obtain the following, more explicit form of the joint distribution of the two strongest values of the STINR process.

Corollary 23. Under the assumptions of Theorem 7, we have

$$
\begin{aligned}
& f_{(k)}^{\prime}\left(z_{1}^{\prime}, \ldots, z_{k}^{\prime}\right) \\
= & \left(\prod_{j=1}^{k}\left(\gamma z_{j}^{\prime}\right)^{-(2 / \beta+1)}\right) \sum_{i=0}^{\left\lceil 1 / \gamma z_{k}^{\prime}+k-1\right\rceil} \frac{(-1)^{i}}{i !} c_{k+i, 2 / \beta, 0} \overline{\mathcal{I}}_{k+i, \beta}\left((W / \gamma) a^{-\beta / 2}\right) \gamma^{k+i} \\
& \times \int_{z_{k}^{\prime}}^{1 / \gamma} \cdots \int_{z_{k}^{\prime}}^{1 / \gamma}\left(\prod_{j=k+1}^{k+i}\left(\gamma \zeta_{j}^{\prime}\right)^{-(2 / \beta+1)}\right)\left(1-\sum_{j=1}^{k}\left(\gamma z_{j}^{\prime}\right)-\sum_{j=k+1}^{k+i}\left(\gamma \zeta_{j}^{\prime}\right)\right)^{2(k+i) / \beta-1} \mathbb{1}\left(\sum_{j=1}^{k}\left(\gamma z_{j}^{\prime}\right)-\sum_{j=k+1}^{k+i}\left(\gamma \zeta_{j}^{\prime}\right)<1\right) d \zeta_{1}^{\prime} \ldots d \zeta_{i}^{\prime},
\end{aligned}
$$

for $z_{1}^{\prime}>z_{2}^{\prime}>\cdots>z_{k}^{\prime}$ and $f_{(k)}^{\prime}\left(z_{1}^{\prime}, \ldots, z_{2}^{\prime}\right)=0$ otherwise.

As a simple example of the application of Proposition 22 or Corollary 23, note that for any $\tau>0$ and $\tau^{\prime}=\tau /(1+\gamma \tau)$

$$
\int_{\tau^{\prime}}^{1 / \gamma} \cdots \int_{\tau^{\prime}}^{1 / \gamma} f_{(k)}^{\prime}\left(z_{1}^{\prime}, \ldots, z_{k}^{\prime}\right) d z_{1}^{\prime}, \ldots d z_{k}^{\prime}=\mathcal{P}^{(k)}(\tau)
$$

is equal to the $k$-coverage probability evaluated in Corollary 19. More developed examples of such applications are studied in the subsequent sections.

Remark 24. Another way for calculating the joint density of the order statistics of the STIR process, using its relations to the two-parameter family of Poisson-Dirichlet processes and a generalized version of the Dickman function, is offered by the result [36, Theorem 5.4]; cf [34] for more details.

\section{B. SINR with interference cancellation and signal combination}

We will now demonstrate how to use the order statistics of the STINR process to express SINR values accounting for general interference cancellation (or management) and signal combination techniques.

Consider a set of $k \geq 1$ strongest signals received by the typical user $\left(Y^{(i)}\right)^{-1}$ (recall that $Y^{(1)}<Y^{(2)}<\ldots$ are order statistics of the propagation process $\{Y\}$ ). Consider a subset $\mathcal{U} \subset[k]:=\{1, \ldots, k\}$ of this set. We define SINR under Interference Cancellation and Signal Combination (ICSC-SINR)

$$
\operatorname{SINR}_{\mathcal{U}, k}=\frac{\sum_{i \in \mathcal{U}}\left(Y^{(i)}\right)^{-1}}{W+\gamma I-\gamma \sum_{j=1}^{k}\left(Y^{(j)}\right)^{-1}},
$$


where signals in $\mathcal{U}$ are combined and interference from all remaining interferers in $[k] \backslash \mathcal{U}$ is canceled. It is easy to see that the ICSC-SINR coverage events can be expressed using the order statistics of the STINR process as follows

$$
\begin{aligned}
& \left\{\operatorname{SINR}_{\mathcal{U}, k}>\tau\right\} \\
& =\left\{\sum_{i \in \mathcal{U}}\left(Y^{(i)}\right)^{-1}>\tau W+\gamma \tau I-\gamma \tau \sum_{j=1}^{k}\left(Y^{(j)}\right)^{-1}\right\} \\
& =\left\{(1+\gamma \tau) \sum_{i \in \mathcal{U}} Z_{(i)}^{\prime}+\gamma \tau \sum_{j \in[k] \backslash \mathcal{U}} Z_{(j)}^{\prime}>\tau\right\} \\
& =\left\{\sum_{i \in \mathcal{U}} Z_{(i)}^{\prime}+\gamma \tau^{\prime} \sum_{j \in[k] \backslash \mathcal{U}} Z_{(j)}^{\prime}>\tau^{\prime}\right\},
\end{aligned}
$$

where, $\tau^{\prime}=\tau /(1+\gamma \tau)$.

Note that the ICSC-SINR coverage (66) says that the ratio of the combined signals to noise plus non-canceled interference is larger than the threshold $\tau$. The probabilities

$$
\mathcal{P}^{(\mathcal{U}, k)}(\tau):=\mathbf{P}\left\{\operatorname{SINR}_{\mathcal{U}, k}>\tau\right\}=\mathbf{P}\left\{\sum_{i \in \mathcal{U}} Z_{(i)}^{\prime}+\gamma \tau^{\prime} \sum_{j \in[k] \backslash \mathcal{U}} Z_{(j)}^{\prime}>\tau^{\prime}\right\}
$$

as function of $\tau$ give the distribution of the ICSC-SINR, thus allowing one to study coverage, spectral efficiency and other characteristics of this channel with ICSC.

The interference cancellation may be imperfect and only reduce the interference power by a factor $\bar{\gamma}<\gamma$; cf [43]. This can be taken into account by replacing $\gamma$ by $\bar{\gamma}$ in (67). Also (66) may yet ignore (practical) conditions under which signal cancellation can be effectively performed. In order to take them into account one can modify (66) as follows

$$
\mathcal{P}_{\mathrm{IC}}^{(\mathcal{U}, k)}(\tau):=\mathbf{P}\left\{\sum_{i \in \mathcal{U}} Z_{(i)}^{\prime}+\mathbb{1}(\mathrm{IC}) \gamma \tau^{\prime} \sum_{j \in[k] \backslash \mathcal{U}} Z_{(j)}^{\prime}>\tau^{\prime}\right\}
$$

where $\mathbb{1}(\mathrm{IC})$ denotes the indicator of a suitable condition for the feasibility of the interference cancellation (IC). Natural conditions for IC can also be expressed in terms of the values $Z_{(i)}^{\prime} i=1, \ldots, k$, and thus $\mathcal{P}_{\mathrm{IC}}^{(\mathcal{U}, k)}$ can be calculated using Proposition 22. Similarly, conditions for the feasibility of the signal combination can be introduced. Here are two examples of natural IC conditions.

1) Successive interference cancellation (SIC): consists in first decoding the strongest interfering signal (among $k$ strongest), subtracting it from the interference, then decoding the second strongest one, and so on. Assume that one can decode a signal if its SINR is larger than some threshold $\epsilon$ (called IC threshold). Denote the indexes of the interfering signals to be decoded and subtracted by $j_{1}, \ldots, j_{k^{\prime}}$, where $k^{\prime}=k-|\mathcal{U}|$, starting from the strongest one. SIC condition can be written then as the superposition of the following conditions.

$$
\begin{array}{r}
Z_{j_{1}}^{\prime}>\epsilon^{\prime} \\
Z_{j_{2}}^{\prime}+\gamma \epsilon^{\prime} Z_{j_{1}}^{\prime}>\epsilon^{\prime} \\
\cdots \\
Z_{j_{k^{\prime}}}^{\prime}+\gamma \epsilon^{\prime} \sum_{l=1}^{k^{\prime}-1} Z_{j_{l}}^{\prime}>\epsilon^{\prime},
\end{array}
$$

where $\epsilon^{\prime}=\epsilon /(1+\gamma \epsilon)$.

2) Independent interference cancellation (IIC): is a weaker condition assuming that all interfering signals to be canceled can be decoded independently: $Z_{(j)}^{\prime}>\epsilon^{\prime}$ for all $j \in[k] \backslash \mathcal{U}$.

\section{Signal to residual interference ratio of the $k$ th strongest signal}

Following Zhang and Haenggi $[25,26]$ let us consider coverage by the $k$ th strongest signal with all $k-1$ stronger signals canceled from the interference, $\mathcal{P}^{(\mathcal{U}, k)}(\tau)$ with $\mathcal{U}=\{k\}$. The following result follows from Proposition 22. Its second statement (case $\gamma \tau>1$ ) with $W=0$ was proved in [26].

Proposition 25. Under the assumptions of Theorem 7 we have

$$
\mathcal{P}^{(\{k\}, k)}(\tau)=\sum_{i=0}^{i_{\max }} \frac{(-1)^{i}}{i !} \int_{0}^{1 / \gamma} \ldots \int_{0}^{1 / \gamma} \mathbb{1}\left(\gamma \tau^{\prime} \sum_{i=1}^{k-1} z_{i}^{\prime}+z_{k}^{\prime}>\tau^{\prime}\right) \mathbb{1}\left(z_{1}^{\prime}>\ldots>z_{k}^{\prime}\right) \mu_{k}^{(k+i)}\left(z_{1}^{\prime}, \ldots, z_{k}^{\prime}\right) d z_{k}^{\prime} \ldots d z_{1}^{\prime},
$$

where the upper summation limit is bounded by

$$
i_{\max }<1 /\left(\gamma \tau^{\prime}\right)-1=1 /(\gamma \tau)
$$


For $\gamma \tau \geq 1$ we have $i_{\max }=0$ and

$$
\begin{aligned}
\mathcal{P}^{(\{k\}, k)}(\tau) & =\frac{\mathcal{I}_{k, \beta}\left((W / \gamma) a^{-\beta / 2}\right)}{\mathcal{I}_{k, \beta}(0)(\gamma \tau)^{2 k / \beta} \Gamma(1+2 k / \beta)(\Gamma(1-2 / \beta))^{k}} \\
& =\frac{2^{k-1} \mathcal{I}_{k, \beta}\left((W / \gamma) a^{-\beta / 2}\right)}{\beta^{k-1}(\gamma \tau)^{2 k / \beta} \Gamma(1+2 k / \beta)(\Gamma(1+2 / \beta))^{k}(\Gamma(1-2 / \beta))^{2 k}}
\end{aligned}
$$

Proof. Using Proposition 22, in order to calculate the probability $\mathcal{P}^{(\{k\}, k)}(\tau)=\mathbf{P}\left\{\gamma \tau^{\prime} \sum_{i=1}^{k-1} Z_{(i)}^{\prime}+Z_{(k)}^{\prime}>\tau^{\prime}\right\}$ one needs to integrate the density $f_{(k)}^{\prime}$ over the domain $\gamma \tau^{\prime} \sum_{i=1}^{k-1} z_{i}^{\prime}+z_{k}^{\prime}>\tau^{\prime}$. A necessary condition for the partial density $\mu_{k}^{\prime(k+i)}$ present in the expansion (62) to be non-null is $\sum_{i=1}^{k-1} z_{i}^{\prime}+(i+1) z_{k}^{\prime}<1 / \gamma$ (cf. the proof of Proposition 22). Combining this condition with the integration domain we obtain $\left(\tau^{\prime}-z_{k}^{\prime}\right) /\left(\gamma \tau^{\prime}\right)+(i+1) z_{k}^{\prime}<1 / \gamma$, which can be rewritten as $z_{k}^{\prime}\left(1+i-1 /\left(\gamma \tau^{\prime}\right)<0\right.$. Since $z_{k}^{\prime} \geq 0$ we have $i<1 /\left(\gamma \tau^{\prime}\right)-1$, which proves (70). The fact that $i_{\max }$ does not depend on the integration variables allows one to interchange the summation and integration as in (69).

For $\gamma \tau \geq 1$ we have $i_{\max }=0$ and the probabilities $\mathcal{P}^{(\{k\}, k)}(\tau)$ can be evaluated as integrals of the $k$ th factorial moment measure of the STINR process over $\gamma \tau^{\prime} \sum_{i=1}^{k-1} z_{i}^{\prime}+z_{k}^{\prime}>\tau^{\prime}$. This is another justification of the equation in (b) in the proof of [26, Theorem 1] and the remaining part of the proof follows the lines presented there, with the contribution of the non-zero noise resulting from the form of the factorial moment measure $M^{\prime(k)}$.

The related result [26, Proposition 2] brings also some approximations for $\mathcal{P}_{S I C}^{(\{k\}, k)}(\tau)$, that is the probability of the above signal-to-residual-interference coverage of the $k$ th strongest signal with SIC condition (with IC threshold $\epsilon=\tau$ ). Exact value of this probability can be obtained restricting the integration domain in (69) as explained in Section V-B1.

In the next section we will consider a somewhat opposite problem, namely, how the coverage by the strongest signal can be improved by removing $k-1$ successive strongest signals from the interference or by combining them with the strongest signal.

\section{Improving the strongest signal by interference cancellation and cooperation}

In this section we consider the following two scenarios:

- When the receiver, being served by the strongest base station, is able to suppress the interference created by the subsequent $k-1$ strongest stations. ${ }^{8}$

- When the subsequent $k-1$ strongest stations can combine their signals with the strongest one.

More specifically, for any $\epsilon, \tau$, with $0<\epsilon<\tau$, and $\epsilon^{\prime}=\epsilon /(1+\gamma \epsilon), \tau^{\prime}=\tau /(1+\gamma \tau)$, we define the following coverage probabilities

$$
\mathcal{P}_{I C}^{(k)}(\tau, \epsilon):=\mathbf{P}\left\{\begin{array}{cl}
\operatorname{SINR}_{\{1\}, k}>\tau & \text { when } Z_{(k)}>\epsilon \\
Z_{(1)}>\tau & \text { otherwise }
\end{array}\right\}=\mathbf{P}\left\{Z_{(1)}^{\prime}+\gamma \tau^{\prime}\left(Z_{(2)}^{\prime}+\ldots+Z_{(k)}^{\prime}\right) \mathbb{1}\left(Z_{(k)}^{\prime}>\epsilon^{\prime}\right)>\tau^{\prime}\right\}
$$

and

$$
\mathcal{P}_{S C}^{(k)}(\tau, \epsilon):=\mathbf{P}\left\{\begin{array}{cl}
\operatorname{SINR}_{[k], k}>\tau & \text { when } Z_{(k)}>\epsilon \\
Z_{(1)}>\tau & \text { otherwise }
\end{array}\right\}=\mathbf{P}\left\{Z_{(1)}^{\prime}+\left(Z_{(2)}^{\prime}+\ldots+Z_{(k)}^{\prime}\right) \mathbb{1}\left(Z_{(k)}^{\prime}>\epsilon^{\prime}\right)>\tau^{\prime}\right\}
$$

Note that $\mathcal{P}_{I C}^{(k)}(\tau, \epsilon)$ is the probability of the coverage by the strongest signal with the cancellation of the interference coming from subsequent $k-1$ strongest signals, whenever these signals individually can be decoded at the SINR level $\epsilon$ and no interference cancellation otherwise. Similarly $\mathcal{P}_{S C}^{(k)}(\tau, \epsilon)$ is the probability of the coverage by the strongest signal combined with $k-1$ subsequent strongest signals, whenever these signals individually can be decoded at the SINR level $\epsilon$ and no signal combination otherwise. Remember that the coverage probability evaluated in Corollary 19 corresponds to $\mathcal{P}(\tau)=\mathcal{P}(k)(\tau)=$ $\mathbf{P}\left\{Z_{(1)}^{\prime}>\tau^{\prime}\right\}$. It is immediately seen that

$$
\mathcal{P}_{S C}^{(k)}(\tau, \epsilon) \geq \mathcal{P}_{I C}^{(k)}(\tau, \epsilon) \geq \mathcal{P}^{(k)}(\tau)
$$

for all $0<\epsilon \leq \tau$. In order to study the difference between the coverage probabilities, denote

$$
\begin{aligned}
& \Delta_{I C}^{(k)}(\tau, \epsilon):=\mathbf{P}\left\{Z_{(1)}^{\prime}+\gamma \tau^{\prime}\left(Z_{(2)}^{\prime}+\ldots+Z_{(k)}^{\prime}\right)>\tau^{\prime} \text { and } \epsilon^{\prime}<Z_{(i)}^{\prime}<\tau^{\prime}, i=1 \ldots k\right\} \\
& \Delta_{S C}^{(k)}(\tau, \epsilon):=\mathbf{P}\left\{Z_{(1)}^{\prime}+\ldots+Z_{(k)}^{\prime}>\tau^{\prime} \text { and } \epsilon^{\prime}<Z_{(i)}^{\prime}<\tau^{\prime}, i=1 \ldots k\right\} .
\end{aligned}
$$

${ }^{8} \mathrm{Cf}$ e.g. $[44,45]$ for methods allowing one to remove from the strong desired signal a weaker interfering one. 
and note that

$$
\begin{aligned}
& \mathcal{P}_{I C}^{(k)}(\tau, \epsilon)=\mathcal{P}(\tau)+\Delta_{I C}^{(k)}(\tau, \epsilon) \\
& \mathcal{P}_{S C}^{(k)}(\tau, \epsilon)=\mathcal{P}(\tau)+\Delta_{S C}^{(k)}(\tau, \epsilon) .
\end{aligned}
$$

We have the following result regarding the increase of the coverage probability of the strongest signal induced by the interference cancellation or signal combination with the subsequent $k-1$ strongest stations.

Proposition 26. Under the assumptions of Theorem 7,

$$
\Delta_{I C}^{(k)}(\tau, \epsilon)=\sum_{i=0}^{i_{\max }} \frac{(-1)^{i}}{i !} \int_{\epsilon^{\prime}}^{\tau^{\prime}} \ldots \int_{\epsilon^{\prime}}^{\tau^{\prime}} \mathbb{1}\left(z_{1}^{\prime}+\gamma \tau^{\prime}\left(z_{2}^{\prime}+\ldots+z_{k}^{\prime}\right)>\tau^{\prime}\right) \mathbb{1}\left(z_{1}^{\prime}>\ldots>z_{k}^{\prime}\right) \mu_{k}^{\prime(k+i)}\left(z_{1}^{\prime}, \ldots, z_{k}^{\prime}\right) d z_{k}^{\prime} \ldots d z_{1}^{\prime}
$$

and

$$
\left.\Delta_{S C}^{(k)}(\tau, \epsilon)=\sum_{i=0}^{i_{\max }} \frac{(-1)^{i}}{i !} \int_{\epsilon^{\prime}}^{\tau^{\prime}} \ldots \int_{\epsilon^{\prime}}^{\tau^{\prime}} \mathbb{1}\left(z_{1}^{\prime}+\ldots+z_{k}^{\prime}\right)>\tau^{\prime}\right) \mathbb{1}\left(z_{1}^{\prime}>\ldots>z_{k}^{\prime}\right) \mu_{k}^{\prime(k+i)}\left(z_{1}^{\prime}, \ldots, z_{k}^{\prime}\right) d z_{k}^{\prime} \ldots d z_{1}^{\prime}
$$

where the upper summation limit is bounded by

$$
i_{\max }<\frac{1}{\gamma \epsilon^{\prime}}-k
$$

Proof. The result follows from Proposition 22. The fact that the integration domain is bounded away from 0 by $\epsilon^{\prime}$ and (63) implies this particular bound on $i_{\max }$. Since it does not depend on the integration variables allows one to interchange the summation and integration as in (74) and (75).

Note that the parameter $\epsilon$ mathematically allows the expansions of $\Delta_{I C}^{(k)}$ and $\Delta_{S C}^{(k)}$ to be finite ones for $i_{\max }<\infty$. We will further numerically study this problem in Section VI-C.

\section{NUMERICAL RESULTS}

\section{A. Setting}

1) Model assumptions: We illustrate our mathematical framework and methods by calculating results for several cellular network models. For all results, we have set $\gamma=1, P S=1$ and $K=1$ and assumed an interference limited network $(W=0)^{9}$ hence the results are scale-invariant (do not depend on $\lambda$ ). Exceptions are results for muti-tier networks, where we will be more specific about $P$ and $\lambda$.

To illustrate the impact of the strength of the path-loss we consider two path-loss exponent values $\beta=3$ and 5 . The network simulations (validating our analytic results) were done in a circular region of radius 10 length units, which was empirically found to be sufficiently large to render so-called edge effects negligible, with number of network simulations being around $10^{5}$.

The SINR thresholds $\tau, \epsilon$ are expressed in $\mathrm{dB}$; i.e. $\tau(\mathrm{dB})=10 \log _{10}(\tau) \mathrm{dB}$.

2) Numerical integration: We employ MATLAB implementation [46] of the quasi-Monte Carlo integration method (based on Sobol points) for integrating the multi-dimensional integrals, which is supported by the theory that says this type of numerical integration generally performs better than regular Monte Carlo integration [47, 48]. From our empirical findings, the evaluation of the $\mathcal{J}_{n, \beta}$ integral is achieved quickly on a standard machine in a matter of seconds (the number of sample points mostly ranges from $10^{3}$ to $10^{4}$ points). The nature of the integral such as (74) and (75) allows the integration with respect to the $z_{i}^{\prime}$ and $v_{i}$ (from $\mathcal{J}_{n, \beta}$ ) to be done in the same computation step, which takes slightly longer to evaluate than $\mathcal{J}_{n, \beta}$. Although, Sobol points perform well in general, it should be noted that the type of quasi-random points can be better chosen if a more thorough analysis of the $\mathcal{J}_{n, \beta}$ integral kernel is performed, but this beyond the scope of this study.

\section{B. Coverage probability}

1) Single-tier network: Figures 1 and 2 present the $k$-coverage probability $\mathcal{P}^{(k)}(\tau)$ for $k=1,2,3$ with $\beta=3$ and 5 , respectively. Recall that $\mathcal{P}^{(k)}(\tau)$ is the tail distribution function of the SINR related to the $k$ th strongest signal.

\footnotetext{
${ }^{9}$ Since including a noise term often makes little difference except for very low network density values
} 




Fig. 1. For $\beta=3, k$-coverage probability $\mathcal{P}^{(k)}(\tau)$ for a single-tier network.

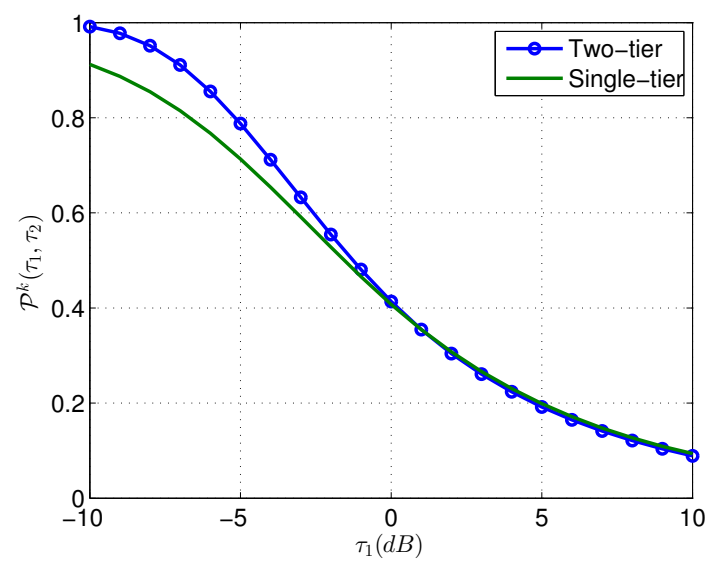

Fig. 3. For $\beta=3$ and for a two-tier network with $\lambda_{1}=\lambda_{2} / 2, P_{1}=$ $100 P_{2} \tau_{2}=1 \mathrm{~dB}$, the 1 -coverage probability $\mathcal{P}^{(1)}$ as a function of $\tau_{1}$ compared to a single-tier network model with equivalent propagation process and constant SINR thresholds.

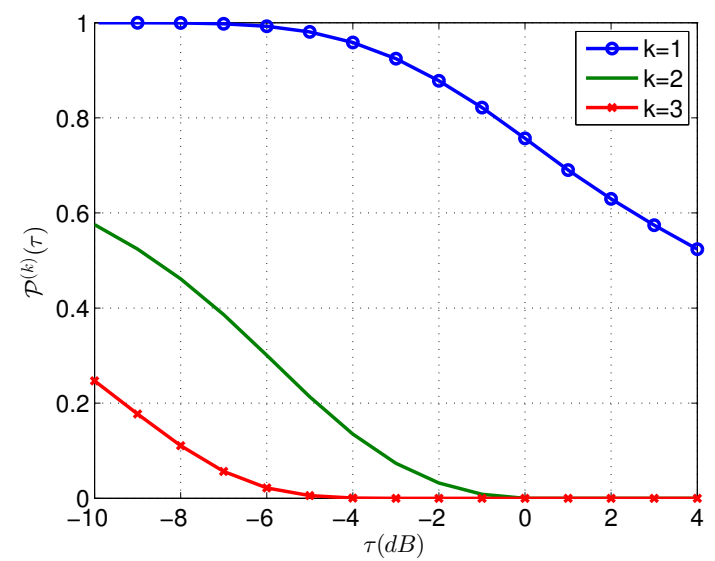

Fig. 2. The same functions as on Figure 1 for $\beta=5$.

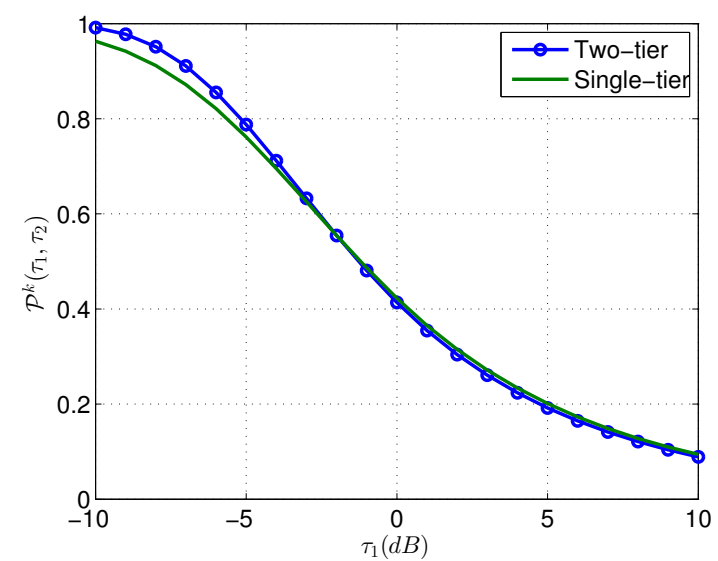

Fig. 4. 1-coverage probability $\mathcal{P}^{(1)}$ for two-tier and single-tier network as on Figure 3 with $\tau_{2}=-2 \mathrm{~dB}$.

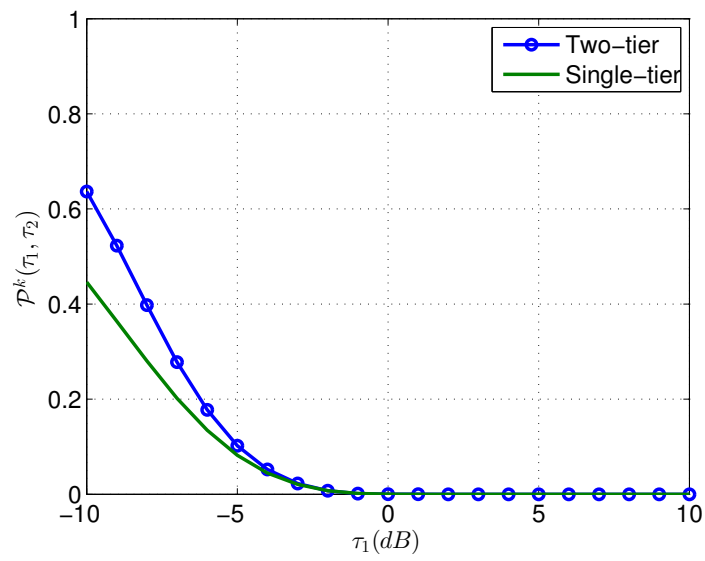

Fig. 5. 2-coverage probability $\mathcal{P}^{(2)}$ for two-tier and one tier-network as on Figure 3 (with $\tau_{2}=-2 \mathrm{~dB}$ ).

2) Two-tier network: We consider two-tier network (cf Section IV-C) with $\lambda_{1}=\lambda_{2} / 2, P_{1}=100 P_{2}$ and $\beta=3$. Considering two values for $\tau_{2}$, namely $\tau_{2}=1(\mathrm{~dB})$ and $\tau_{2}=-2(\mathrm{~dB})$, on Figures $3,4,5$ we plot 1- and 2-coverage probability $\mathcal{P}^{(k)}$, $k=1,2$ as functions of $\tau_{1}$. The parameters of the two-tier network are based on previous results [14, Fig.7.]. For 1-coverage probability, the results agree with those in [14], thus validating the generalized symmetric sum method in the multi-tier setting.

For comparison purposes, on Figures 3, 4, 5 we consider also a single-tier network model with intensity $\lambda^{*}$ given by (54) i.e., $\lambda^{*}=\lambda_{1} P_{1}^{2 / \beta}+\lambda_{2} P_{2}^{2 / \beta}$ (which gives equivalent propagation and SINR processes) for which we calculate the similar 


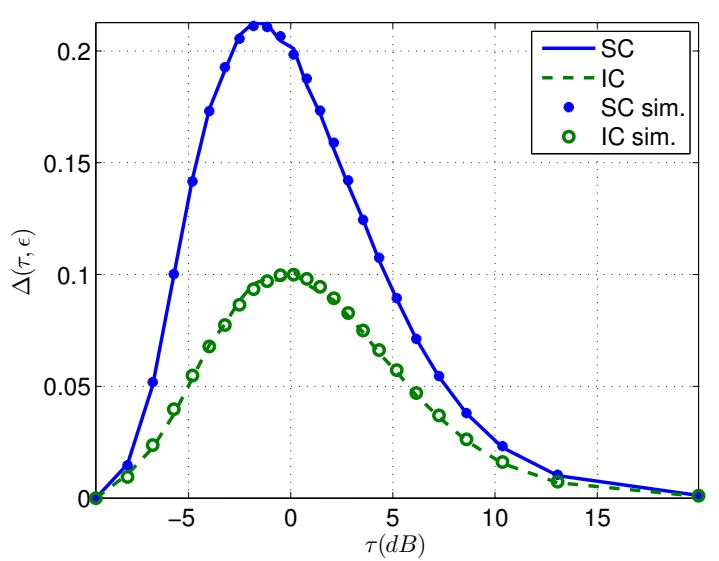

Fig. 6. For $\beta=3$, the increase of the coverage probability of the strongest signal induced by the interference cancellation $\Delta_{I C}^{(2)}(\tau, \epsilon)$ or signal combination $\Delta_{S C}^{(2)}(\tau, \epsilon)$ with the second strongest station assuming $\epsilon^{\prime}=0.1$ (or $\epsilon=-9.5424 \mathrm{~dB}$ ).

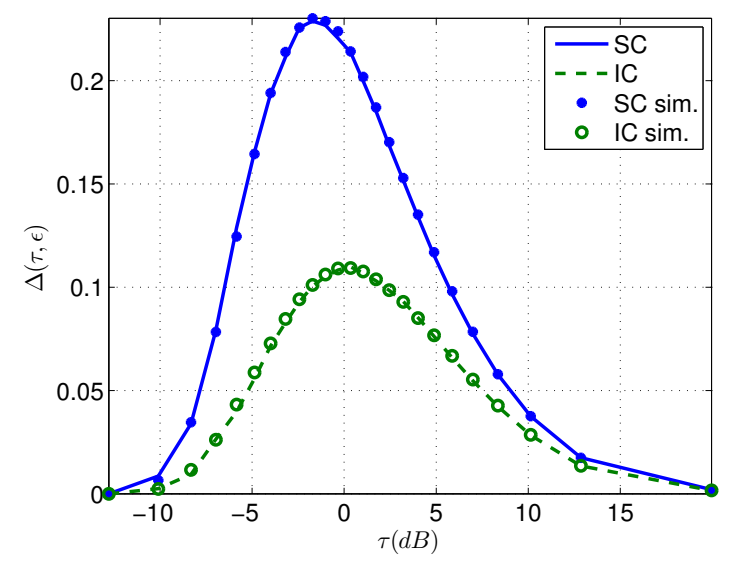

Fig. 8. The same functions as on Figure 6 with $\beta=3$ and $\epsilon^{\prime}=0.05$ (or $\epsilon=-12.7875 \mathrm{~dB}$ ).

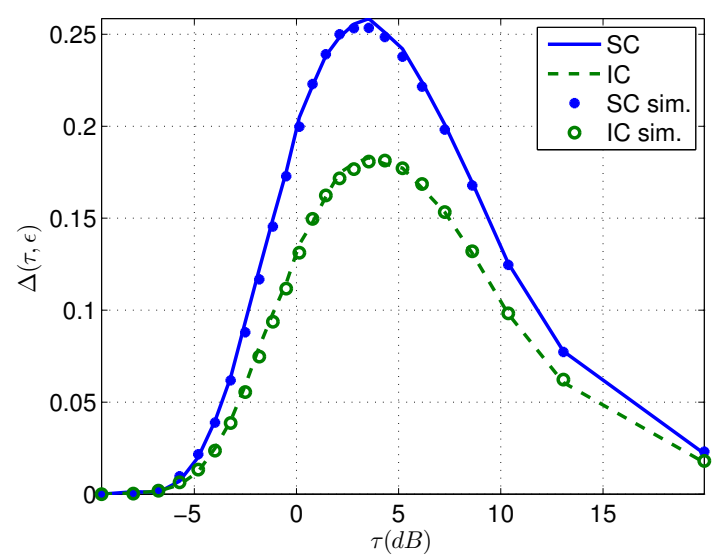

Fig. 7. The same functions as on Figure 6 for $\beta=5$.

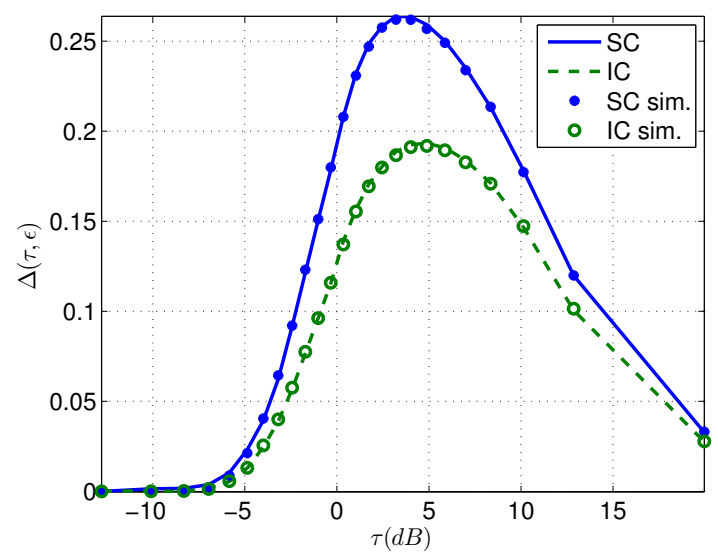

Fig. 9. The same functions as on Figure 6 with $\beta=5$ and $\epsilon^{\prime}=0.05$ (or $\epsilon=-12.7875 \mathrm{~dB}$ ).

$k$-coverage probabilities with constant SINR thresholds $\tau=\mathbf{E}\left[T^{*}\right]=\left(\tau_{1} \lambda_{1} P_{1}^{2 / \beta}+\tau_{2} \lambda_{2} P_{2}^{2 / \beta}\right) / \lambda^{*}$, equal to the mean of the random SINR threshold mark in the truly equivalent model. Note that for the considered parameter values, this model offers quite reasonable approximation of the original two-tier one, in particular for $\tau>1$ (although visually the corresponding plots almost coincide, it remains an approximation). It would be interesting to see under which conditions the above observation can be generalized, i.e., when one can approximate a multi-tier model with the single-tier model generating the equivalent propagation process and having averaged $\tau$ vales.

\section{Coverage with interference cancellation and cooperation}

On Figures 6, 7, 8 and 9 we study the increase of the coverage probability of the strongest signal induced by the interference cancellation $\Delta_{I C}^{(2)}(\tau, \epsilon)$ or signal combination $\Delta_{S C}^{(2)}(\tau, \epsilon)$ with the second strongest station. We consider two values of the path-loss exponent $\beta=3$ and $\beta=5$ and the decoding threshold (for cancellation and combination) $\epsilon=-9.5424 \mathrm{~dB}$ and $-12.7875 \mathrm{~dB}$.

The plots of the probabilities reveal that the increase of coverage probability is largest around the threshold $\tau=1(0 \mathrm{~dB})$. Also, as the path-loss exponent $\beta$ increases the difference between the gain under IC and SC decreases (cf. Figure 6 to 9). This is explained by realizing that the second largest interfering signal, which is removed under IC, weakens as $\beta$ increases.

Recall that the decoding threshold $\epsilon$ mathematically allows the expansion of $\Delta_{I C}^{(k)}$ and $\Delta_{S C}^{(k)}$ in (74) and (75) to be finite ones, hence they are complete expressions when some $i_{\max }<\infty$ terms are included. Although as $\epsilon$ decreases more terms are needed, not all the integral terms are necessary to gain a good approximation (Figure 10 and 11). Numerical evidence confirms the mathematical intuition that as $\epsilon$ decreases the integral kernel approaches the singularity at $t_{i}=0$ (for some integer $i \in[n]$ ), hence the peak of the resulting integral increases.

Results for $\epsilon^{\prime}=0.05$ (i.e., $\epsilon=-12.7875 \mathrm{~dB}$ ) were obtained with relative ease and speed, but for smaller $\epsilon^{\prime}$, it becomes computationally impracticable. 


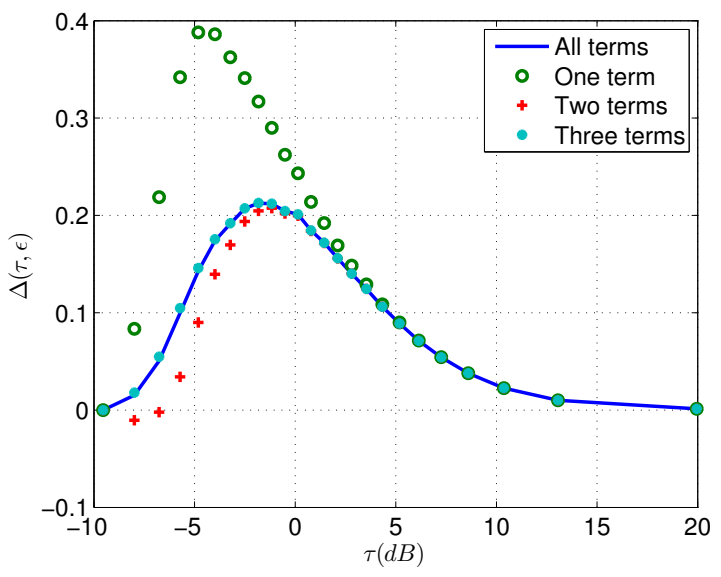

Fig. 10. For $\beta=3$, the complete (10-term) expression and 1, 2, and 3-term approximations for the $\Delta_{S C}^{(2)}$ probability with $\epsilon^{\prime}=0.1$ (or $\epsilon=-9.5424 \mathrm{~dB})$.

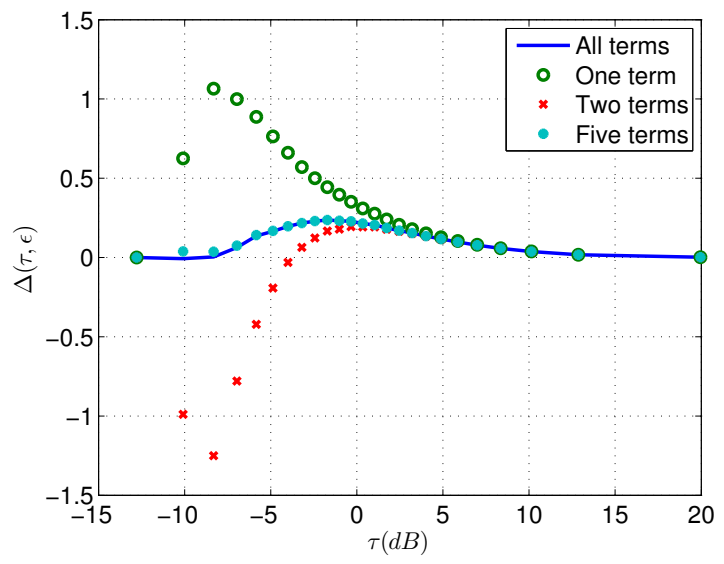

Fig. 11. For $\beta=3$, the complete (20-term) expression and 1, 2, and 5-term approximations for the $\Delta_{S C}^{(2)}$ probability for a heterogeneous network under two base station cooperation with $\epsilon^{\prime}=0.05$ (or $\epsilon=$ $-12.7875 \mathrm{~dB})$.

Broadly speaking, the more integration points are needed for larger $\beta$ and smaller $\epsilon$. The effect of $\beta$ probably stems from the Sobol points not sampling the integral kernel adequately, which could be improved by way of a suitable importance sampling. That said, for $\beta=5$ and $\epsilon^{\prime}=0.05$ the $\Delta$ probability plots (Figure 9) were still obtained fast (in seconds) except for small $\tau$ which required slightly more time.

\section{FUTURE DIRECTIONS AND CONCLUSIONS}

We propose some model and technique extensions, discuss their feasibility and conclude the work.

\section{A. General functions of the SINR process via the factorial-moment expansion}

We used the factorial moment measures of the SINR process $\Psi$ to derive expressions for $k$-coverage probabilities via the famous inclusion-exclusion principle. More general characteristics of the coverage number can be obtained directly using Schuette-Nesbitt formula (which we recall for completeness in Appendix F, see also [42]). Using a result by Handa [36, Lemma 5.3], we also demonstrated how to calculate the joint densities of the order statistics of the SINR process using its factorial moment measures. It turns out that all these results can be seen as instances of some more general expression allowing one to calculate (at least theoretically) the expectations of general functions $\phi$ of simple point processes by means of a (finite or infinite) Taylor-like expansion. The terms of this expansion are integrals of some kernels (entirely characterized by $\phi$ ) with respect to the factorial moment measures of the considered point process, $\mathrm{cf}$ [5].

More specifically, under appropriate convergence conditions (whose presentation is beyond the scope of this paper) one can write the expectation of a general function $\phi$ of the (say) STINR point process $\Psi^{\prime}$ as

$$
\mathbf{E}\left[\phi\left(\Psi^{\prime}\right)\right]=\phi(\emptyset)+\sum_{n=1}^{\infty} \int_{0}^{1 / \gamma} \ldots \int_{0}^{1 / \gamma} \phi_{t_{1}^{\prime}, \ldots, t_{n}^{\prime}} \mu^{\prime(n)}\left(t_{1}^{\prime}, \ldots, t_{n}^{\prime}\right) d t_{n}^{\prime} \ldots d t_{1}^{\prime},
$$

where $\mu^{\prime(n)}\left(t_{1}^{\prime}, \ldots t_{n}^{\prime}\right)$ is the density of the $n$th factorial moment measure of the STINR process given in Corollary $10, \phi(\emptyset)$ denotes the value of the function $\phi$ evaluated on the empty configuration of points (no points) and

$$
\begin{aligned}
\phi_{t_{1}^{\prime}} & =\phi\left(\left\{t_{1}^{\prime}\right\}\right)-\phi(\emptyset) \\
\phi_{t_{1}^{\prime}, t_{2}^{\prime}} & =\frac{1}{2}\left(\phi\left(\left\{t_{1}^{\prime}, t_{2}^{\prime}\right\}\right)-\phi\left(\left\{t_{1}^{\prime}\right\}\right)-\phi\left(\left\{t_{2}^{\prime}\right\}\right)+\phi(\emptyset)\right) \\
\ldots & \\
\phi_{t_{1}^{\prime}, \ldots, t_{n}^{\prime}} & =\frac{1}{n !} \sum_{k=0}^{n}(-1)^{n-k} \sum_{\substack{t_{i_{1}}^{\prime}, \ldots, t_{i}^{\prime} \\
\text { distinct }}} \phi\left(\left\{t_{i_{1}}^{\prime}, \ldots, t_{i_{k}}^{\prime}\right\}\right) .
\end{aligned}
$$

For example, setting

$$
\phi\left(\left\{t_{1}^{\prime}, \ldots\right\}\right)=\mathbb{1}\left(\max _{i \geq 1} t_{i}^{\prime}>\tau^{\prime}\right)
$$

and applying (77) one obtains (42) with $k=1$ in the case of constant STINR threshold $\tau^{\prime}$. More generally, using (77) with

$$
\phi\left(\left\{t_{1}^{\prime}, \ldots\right\}\right)=\prod_{k}\left(h\left(t_{k}^{\prime}\right) \mathbb{1}\left(t_{k}^{\prime}>\tau^{\prime}\right)+\mathbb{1}\left(t_{k}^{\prime} \leq \tau^{\prime}\right)\right)
$$


one obtains the following expansion of the probability generating function (cf [29, Eq. 5.5.4]) of the SINR process

$$
\mathbf{E}\left[\prod_{Z^{\prime} \in \Psi^{\prime}}\left(h\left(Z^{\prime}\right) \mathbb{1}\left(Z^{\prime}>\tau^{\prime}\right)+\mathbb{1}\left(Z^{\prime} \leq \tau^{\prime}\right)\right)\right]=1+\sum_{n=1}^{n_{\max }} \frac{1}{n !} \int_{\tau^{\prime}}^{1 / \gamma} \int_{\tau^{\prime}}^{1 / \gamma} \prod_{k=1}^{n}\left(h\left(t_{k}^{\prime}\right)-1\right) \mu^{\prime(n)}\left(t_{1}^{\prime}, \ldots, t_{n}^{\prime}\right) d t_{n}^{\prime} \ldots d t_{1}^{\prime}
$$

where the structure of our point process imposes $n_{\max }<1 /\left(\gamma \tau^{\prime}\right)$, for any bounded function $h(\cdot)$. This expansion can be easily transformed to the one for STINR via the mapping (10). See [29, Sec. 5.5] for other classical moment expansion results.

Thus the factorial-moment expansion provides a way for calculating various SINR-based quantities, thus opening a range of other possible models coupled with analytic methods.

\section{B. Varying path-loss exponent model}

For tractability we used a fixed path-loss exponent $\beta$ and $K$ throughout this work. However, an argument can be made based on the Hata-like path-loss models that both constants incorporate base station height [49, Section 2.7.3]. Consequently, more adequate heterogeneous network models may require $\beta$ and $K$ to be random marks of base stations. Extension of our model to random $K$ is straightforward. Considering random $\beta$ is more complicated. It was recently shown that in terms of propagation losses, a Poisson network with random $\beta$ and constant base station density $\lambda$ is equivalent to some network with constant exponents and an isotropic (but not power-law) density [7]. Assuming such density increases the complexity of the factorial moment integrals, but they may remain amenable, particularly to numerical means. Multi-tier models with values of $\beta$ depending on tiers have been considered in $[16,20]$.

\section{Other path-loss functions}

Path-loss functions other than the commonly used (singular) power-law function $\ell$ have been previously proposed, including exponential types and modified power-laws with the singularity removed (for example, $\ell(|x|)=\min \left(1,(|x|)^{\beta}\right)$. Unfortunately, two immediate drawbacks arise when employing other path-loss functions. The propagation invariance (Lemma 1) would no longer hold ${ }^{10}$, hence the factorial moments would need to be calculated for different distributions of propagation effects. Furthermore, the algebraic tractability in our work would quickly diminish; for example, the change of variables used (in Section B) would, most probably, not work in simplifying the integrals.

\section{Non-Poisson base station configurations}

Our derivation of the explicit expressions of $\mathcal{S}_{n}$, via the factorial moment measures of the SINR process, hinges heavily upon the tractable nature of the Poisson process (in particular, Slivnyak's theorem). Despite this, the $M^{(n)}$-dependent $\mathcal{S}_{n}$ terms and related expressions introduced in Section IV-B do not rely upon the Poisson assumption. More specifically, assume a simple point process $\Phi=\{X\}$ representing the base stations, which gives rise to a corresponding STINR process $\Psi^{\prime}=\left\{Z^{\prime}\right\}$. Then deriving the $\mathcal{S}_{n}$ terms is again equivalent to the task of finding the corresponding factorial moment measures of $\left\{Z^{\prime}\right\}$, which in turn immediately give those of $\{Z\}$.

However, a non-Poisson base station configuration would require considerably more work due to Slivnyak's theorem no longer being applicable. Furthermore, we have leveraged propagation invariance considerably, which flows directly from the Poisson assumption. Without it, the factorial moment measure of $\left\{Z^{\prime}\right\}$ would need to be calculated for different distributions of propagation effects given that the propagation invariance would not hold. The propagation invariance also granted us the ability to create an equivalent and more tractable network $\Phi^{*}$ used in Proposition 14. A cellular network model with base stations positioned according to a Ginibre process was recently considered in $[50,51]$ where an expression for the SINR coverage probability by the closest base station was derived assuming Rayleigh fading (which does not impact the choice of the serving station, as in [21], [18, Section IV.C] for the Poisson model).

\section{E. Conclusions}

We have presented a novel framework for studying general down-link characteristics in a heterogeneous cellular network. It is based on the explicit evaluation of the factorial moment measures of the point process formed by the SINR values observed by a typical user with respect to all network stations. They allow one, in particular, to express the finite dimensional distributions of the ordered vectors of $k$ strongest SINR values (SINR order statistics). We have demonstrated the benefits of this approach by extending existing results and deriving new ones regarding the SINR coverages probabilities, in particular pertaining to the usage of signal combination and interference cancellation techniques.

\footnotetext{
${ }^{10}$ For certain path-loss functions, less elegant invariance results may exist where propagation processes rely upon two or more propagation moments.
} 


\section{APPENDIX}

A. Remarks on $\mathcal{J}_{n, \beta}$

The integral $\mathcal{J}_{n, \beta}\left(x_{1}, \ldots, x_{n}\right)$ can be written as

$$
\mathcal{J}_{n, \beta}\left(x_{1}, \ldots, x_{n}\right)=\frac{1}{n} \sum_{j=1}^{n} \int_{[0,1]^{n-1}} \frac{\prod_{i=1}^{n-1} v_{i}^{i(2 / \beta+1)-1}\left(1-v_{i}\right)^{2 / \beta}}{\prod_{i \neq j}\left(x_{i}+\eta_{i}\right)} d v_{1} \ldots d v_{n-1}
$$

For $n=1$ the integral $\mathcal{J}_{1, \beta}\left(x_{1}\right)=1$ and for $n=2$ the integral reduces to a sum of two integrals of the same type

$$
\mathcal{J}_{2, \beta}\left(x_{1}, x_{2}\right)=\frac{1}{2} \int_{0}^{1} v_{1}^{2 / \beta}\left(1-v_{1}\right)^{2 / \beta}\left[\frac{1}{\left(x_{1}+v_{1}\right)}+\frac{1}{\left(x_{2}+1-v_{1}\right)}\right] d v_{1} \text {. }
$$

The general solution to this integrals is

$$
\int_{0}^{1} \frac{v^{2 / \beta}(1-v)^{2 / \beta}}{x+v} d v=\frac{1}{x} B(2 / \beta+1,2 / \beta+1){ }_{2} F_{1}(1,2 / \beta+1 ; 2(2 / \beta+1) ;-1 / x),
$$

where is ${ }_{2} F_{1}$ a hypergeometric function [52, Equation 15.6.1].

Given the definition of $\mathcal{J}_{n, \beta}$, one can write

$$
\mathcal{J}_{n, \beta}\left(x_{1}, \ldots, x_{n}\right)=\frac{1}{n} \mathbf{E}\left[H\left(x_{1}, \ldots, x_{n}, \widetilde{V}_{1}, \ldots, \widetilde{V}_{n}\right)\right] \prod_{i=1}^{n-1} B(1+2 / \beta, i(2 / \beta+1)),
$$

where the permutation-invariant function

$$
\begin{aligned}
H\left(x_{1}, \ldots, x_{n}, \widetilde{V}_{1}, \ldots, \widetilde{V}_{n}\right) & =\sum_{j=1}^{n}\left(x_{j}+\widetilde{V}_{j}\right) \prod_{i=1}^{n}\left(x_{i}+\widetilde{V}_{i}\right)^{-1} \\
& =\left(\sum_{j=1}^{n} x_{j}+1\right) \prod_{i=1}^{n}\left(x_{i}+\widetilde{V}_{i}\right)^{-1}
\end{aligned}
$$

and $\widetilde{V}_{i}$ are beta random variables with distributions $B(2 / \beta+1, i(2 / \beta+1))$. This interpretation may offer an alternative way for numerically evaluating $\mathcal{J}_{n, \beta}$; for example, simulating $V_{i}$ and estimating the mean of $H$. Furthermore, as remarked earlier there seems to be a deeper connection between the STINR process and Poisson-Dirichlet processes owing to them both being connected to beta variables of this type, cf. [36].

\section{B. Proof of Theorem 7}

In view of the propagation invariance Lemma 1, without loss of generality we can assume $K=1$ and $(P S)$ having exponential distribution with mean one and replace $\lambda$ with $a /(\pi \Gamma(1+2 / \beta))$, where $a$ is given by (4) (note that $\Gamma(2 / \beta+1)$ is the $2 / \beta$ th moment of exponential variable of mean one).

The definition of $\Psi^{\prime}=\left\{Z^{\prime}\right\}$ and its factorial moment measure (12) give via the Campbell's formula (for factorial moment measures) and Slivnyak theorem (for example, see [31])

$$
M^{\prime(n)}\left(t_{1}^{\prime}, \ldots t_{n}^{\prime}\right)=a^{n} \int_{\left(\mathbb{R}^{2}\right)^{n}} \mathbf{E}\left(\prod_{j=1}^{n} \mathbb{1}\left(\frac{E_{j} t_{j}^{\prime}}{W+\gamma\left[I+\sum_{i=1}^{n} E_{i} t_{i}^{\prime}\right]}>t_{j}^{\prime}\right)\right) d x_{1} \ldots d x_{n}
$$

where $E_{i}:=\left(P_{i} S_{i}\right) /\left(\ell\left(r_{i}\right) t_{i}^{\prime}\right)$. The integral reduces to

$$
\begin{aligned}
M^{\prime(n)}\left(t_{1}^{\prime}, \ldots t_{n}^{\prime}\right) & =(2 \pi a)^{n} \int_{\left(\mathbb{R}_{+}\right)^{n}} \mathbf{E}\left(\prod_{j=1}^{n} \mathbb{1}\left(\frac{E_{j}}{W+\gamma\left[I+\sum_{i=1}^{n} E_{i} t_{i}^{\prime}\right]}>1\right) r_{1} d r_{1} \ldots r_{n} d r_{n}\right. \\
& =(2 \pi a)^{n} \int_{\left(\mathbb{R}_{+}\right)^{n}} \mathbf{P}\left(\frac{\min \left[E_{1}, \ldots, E_{n}\right]}{W+\gamma\left[I+\sum_{i=1}^{n} E_{i} t_{i}^{\prime}\right]}>1\right) r_{1} d r_{1} \ldots r_{n} d r_{n} .
\end{aligned}
$$


Since $P_{i} S_{i}$ are exponentially distributed with unit means, then $E_{i}$ are also exponentially distributed with means $1 / \mu_{i}=$ $1 /\left(t_{i}^{\prime} \ell\left(r_{i}\right)\right)$, and let

$$
\hat{E}_{M}=\min \left(E_{1}, \ldots, E_{n}\right)
$$

which is another exponential variable with parameter $\mu_{M}=\sum_{i=1}^{n} \mu_{i}$. Let

$$
D=\sum_{i=1}^{n} E_{i} t_{i}^{\prime}-\hat{E}_{M} \sum_{i=1}^{n} t_{i}^{\prime}=\sum_{i=1}^{n}\left(E_{i}-\hat{E}_{M}\right) t_{i}^{\prime}
$$

Note, $\hat{E}_{M}=E_{j}$ with probability $\mu_{j} /\left(\sum_{i=1}^{n} \mu_{i}\right)$. Given this event $E_{i}-E_{j}(i \neq j)$ are conditionally independent, exponential variables with parameters $\mu_{i}$, and independent of $\hat{E}_{M}$. (This is the well known memoryless property of the exponential distribution.) Consequently $\hat{E}_{M}$ and $D$ are independent and the latter has the following mixed exponential distribution

$$
\mathbf{P}(D \leq d)=\frac{1}{\sum_{i=1}^{n} \mu_{i}}\left[\sum_{j=1}^{n} \mu_{j} \mathbf{P}\left(\sum_{i \neq j} E_{i} t_{i}^{\prime} \leq d\right)\right] .
$$

Observe that

$$
\begin{aligned}
\mathbf{P}\left(\frac{\min \left(E_{1}, \ldots, E_{n}\right)}{W+\gamma\left[I+\sum_{i=1}^{n} E_{i} t_{i}^{\prime}\right]}>1\right) & =\mathbf{P}\left(\frac{\hat{E}_{M}}{W+\gamma\left[I+D+\hat{E}_{M} \sum_{i=1}^{n} t_{i}^{\prime}\right]}>1\right) \\
& =\mathbf{P}\left(\frac{\hat{E}_{M}}{W+\gamma[I+D]}>\hat{T}_{n} / \gamma\right) \\
& =\mathbf{P}\left(\frac{\hat{E}_{M}}{W / \gamma+I+D}>\hat{T}_{n}\right),
\end{aligned}
$$

where

$$
\hat{T}_{n}:=\hat{T}_{n}\left(t_{1}, \ldots, t_{n}\right)=\gamma /\left(1-\gamma \sum_{i=1}^{n} t_{i}^{\prime}\right)
$$

Given that $W, I, D$, and $\hat{E}_{M}$ are mutually independent, and that $\hat{E}_{M}$ is exponentially distributed, hence

$$
\mathbf{P}\left(\frac{\hat{E}_{M}}{W / \gamma+I+D}>\hat{T}_{n}\right)=\mathcal{L}_{W / \gamma}\left(\mu_{M} \hat{T}_{n}\right) \mathcal{L}_{I}\left(\mu_{M} \hat{T}_{n}\right) \mathcal{L}_{D}\left(\mu_{M} \hat{T}_{n}\right),
$$

which is a product of three Laplace transforms. The first transform

$$
\mathcal{L}_{W / \gamma}(\xi)=e^{-\xi W / \gamma}
$$

and the second (see equation 2.25 in [40])

$$
\mathcal{L}_{I}(\xi)=e^{-a \xi^{2 / \beta} \pi C^{\prime}(\beta) / K^{2}}
$$

immediately follow. Given expression (78), then the Laplace transform of a general exponential variable and the convolution theorem imply that the distribution of $D$ has the transform

$$
\mathcal{L}_{D}(\xi)=\frac{\prod_{i=1}^{n} \mu_{i}}{\sum_{i=1}^{n} \mu_{i}}\left[\sum_{j=1}^{n} \frac{1}{\left(\prod_{i \neq j}\left[\mu_{j}+t_{j}^{\prime} \xi\right]\right)}\right] .
$$

After substituting the explicit path-loss function (1) with $K=1$, which will be recovered later, and some algebra we arrive at

$$
\begin{aligned}
& \mathbf{P}\left(\frac{\hat{E}_{M}}{W / \gamma+I+D}>\hat{T}_{n}\right) \\
& \quad=\frac{\left(\prod_{i=1}^{n} t_{i}^{\prime} r_{i}^{\beta} e^{-(W / \gamma) \hat{T}_{n} t_{i}^{\prime} r_{i}^{\beta}}\right) e^{-a\left(\hat{T}_{n} \sum_{i=1}^{n} t_{i}^{\prime} r_{i}^{\beta}\right)^{2 / \beta} \pi C^{\prime}(\beta)}}{\sum_{i=1}^{n} t_{i}^{\prime} r_{i}^{\beta}} \sum_{j=1}^{n}\left(\frac{1}{\prod_{i \neq j}\left[t_{i}^{\prime} r_{i}^{\beta}+t_{i} \hat{T}_{n} \sum_{k=1}^{n} t_{k}^{\prime} r_{k}^{\beta}\right]}\right) .
\end{aligned}
$$


Hence, the integral

$$
\begin{aligned}
& M^{\prime(n)}\left(t_{1}^{\prime}, \ldots, t_{n}^{\prime}\right) \\
& =(2 \pi a)^{n} \int_{\left(\mathbb{R}_{+}\right)^{n}} \frac{\left(\prod_{i=1}^{n} t_{i}^{\prime} r_{i}^{\beta} e^{-(W / \gamma) \hat{T}_{n} t_{i}^{\prime} r_{i}^{\beta}}\right)}{\sum_{i=1}^{n} t_{i}^{\prime} r_{i}^{\beta}} e^{-a\left(\hat{T}_{n} \sum_{i=1}^{n} t_{i}^{\prime} r_{i}^{\beta}\right)^{2 / \beta} \pi C^{\prime}(\beta)} \sum_{j=1}^{n}\left(\frac{1}{\prod_{i \neq j}\left[t_{i}^{\prime} r_{i}^{\beta}+t_{i}^{\prime} \hat{T}_{n} \sum_{k=1}^{n} t_{k}^{\prime} r_{k}^{\beta}\right]}\right) r_{1} d r_{1} \ldots r_{n} d r_{n},
\end{aligned}
$$

with the variable change $s_{i}:=r_{i} t_{i}^{1 / \beta}\left(a \hat{T}_{n}^{2 / \beta} \pi C^{\prime}(\beta)\right)^{1 / 2}$ reduces to

$$
\begin{aligned}
& M^{\prime(n)}\left(t_{1}^{\prime}, \ldots, t_{n}^{\prime}\right) \\
& =\frac{2^{n} \hat{T}_{n}^{-2 n / \beta}}{\left(C^{\prime}(\beta)\right)^{n}} \int_{\left(\mathbb{R}_{+}\right)^{n}} \frac{\left(\prod_{i=1}^{n} t_{i}^{\prime-2 / \beta} s_{i}^{\beta+1} e^{-(W / \gamma)\left(a \pi C^{\prime}(\beta)\right)^{-\beta / 2} s_{i}^{\beta}}\right)}{\sum_{i=1}^{n} s_{i}^{\beta}} e^{-\left(\sum_{i=1}^{n} s_{i}^{\beta}\right)^{2 / \beta}} \sum_{j=1}^{n}\left(\frac{1}{\prod_{i \neq j}\left[s_{i}^{\beta}+t_{i}^{\prime} \hat{T}_{n} \sum_{k=1}^{n} s_{k}^{\beta}\right]}\right) d s_{1} \ldots d s_{n} .
\end{aligned}
$$

We introduce a change of variables inspired by the $n$-dimensional spherical coordinates

$$
\begin{aligned}
s_{1} & =u\left[\sin \theta_{1} \sin \theta_{2} \ldots \sin \theta_{n-1}\right]^{2 / \beta} \\
s_{2} & =u\left[\cos \theta_{1} \sin \theta_{2} \ldots \sin \theta_{n-1}\right]^{2 / \beta} \\
s_{3} & =u\left[\cos \theta_{2} \sin \theta_{3} \ldots \sin \theta_{n-1}\right]^{2 / \beta} \\
& \ldots \\
s_{n} & =u\left[\cos \theta_{n-1}\right]^{2 / \beta} .
\end{aligned}
$$

Note that $u^{\beta}=\sum_{i=1}^{n} s_{i}^{\beta}$ and $\prod_{i=1}^{n} s_{i}=u^{n}\left[\prod_{i=1}^{n} q_{i}\right]^{2 / \beta}$ where we use the shorthand $q_{i}=q_{i}\left(\theta_{1}, \ldots, \theta_{n}\right):=\left(s_{i} / u_{i}\right)^{\beta / 2}$ for $u_{i}>0$. For $\beta=2$, we obtain $n$-dimensional spherical coordinates with a Jacobian

$$
\hat{J}\left(\hat{u}, \hat{\theta}_{1}, \ldots, \hat{\theta}_{n}\right)=\hat{u}^{n-1} \prod_{i=1}^{n-1} \sin ^{i-1} \hat{\theta}_{i}
$$

while our variables have the Jacobian

$$
J\left(u, \theta_{1}, \ldots, \theta_{n}\right)=\left(\frac{2}{\beta}\right)^{n-1} \hat{J}\left(u, \theta_{1}, \ldots, \theta_{n}\right)\left[\prod_{i=1}^{n-1} \sin ^{i} \theta_{i} \cos \theta_{i}\right]^{2 / \beta-1}
$$

Denote $z:=(W / \gamma)(a \Gamma(1-2 / \beta))^{-\beta / 2}$. The change of variable renders the integral as

$$
\begin{aligned}
& M^{\prime(n)}\left(t_{1}, \ldots, t_{n}\right) \\
& =\frac{2^{n} \hat{T}_{n}^{-2 n / \beta}}{\left(C^{\prime}(\beta)\right)^{n}} \int_{\left(\mathbb{R}_{+}\right)^{n}} \frac{\left(\prod_{i=1}^{n} t_{i}^{\prime-2 / \beta} s_{i}^{\beta+1} e^{-z s_{i}^{\beta}}\right)}{\sum_{i=1}^{n} s_{i}^{\beta}} e^{-\left(\sum_{i=1}^{n} s_{i}^{\beta}\right)^{2 / \beta}} \sum_{j=1}^{n}\left(\frac{1}{\prod_{i \neq j}\left[s_{i}^{\beta}+t_{i}^{\prime} \hat{T}_{n} \sum_{k=1}^{n} s_{k}^{\beta}\right]}\right) d s_{1} \ldots d s_{n} \\
& =\frac{2^{n} \hat{T}_{n}^{-2 n / \beta}}{\left(C^{\prime}(\beta)\right)^{n}}\left(\frac{2}{\beta}\right)^{n-1} \prod_{i=1}^{n} t_{i}^{\prime-2 / \beta} \int_{0}^{n-1} u^{2 n-1} e^{-\left(u^{2}+z u^{\beta}\right)} d u \int_{[0, \pi / 2]^{n-1}}^{n} \sum_{j=1}^{n} \frac{\left.\prod_{i=1}^{i} \theta_{i} \cos \theta_{i}\right]^{4 / \beta+1}\left[\sin \theta_{i}\right]^{i-1}}{\prod_{i \neq j}\left[q_{i}^{2}+t_{i}^{\prime} \hat{T}_{n}\right]} d \theta_{1} \ldots d \theta_{n-1} .
\end{aligned}
$$

We then substitute $v_{i}=\sin ^{2} \theta_{i}$, and define $\eta_{i}$ and $\hat{t}_{i}$ accordingly. We define $\mathcal{J}_{n}\left(x_{1}, \ldots, x_{n}\right)$ to be analogous (to equation (15) in [18]),

$$
\mathcal{J}_{n, \beta}\left(x_{1}, \ldots, x_{n}\right)=\frac{1}{n} \int_{[0,1]^{n-1}} \sum_{j=1}^{n} \frac{\prod_{i=1}^{n-1} v_{i}^{i(2 / \beta+1)-1}\left(1-v_{i}\right)^{2 / \beta}}{\prod_{i \neq j}\left(x_{i}+\eta_{i}\right)} d v_{1} \ldots d v_{n-1},
$$

but observe, since $\sum_{j=1}^{n} \eta_{j}=1$, that the above reduces to integral (16), completing the proof. 


\section{Partial densities of $M^{\prime(n)}$}

We will now detail how to calculate the partial derivatives $\frac{\partial^{k}}{\partial t_{1}^{\prime}, \ldots \partial t_{k}^{\prime}}$ of $M^{\prime(n)}$, for $k \leq n$ and thus obtain explicit expressions for $\mu_{k}^{\prime k+i}$. Note first that the integrals $\mathcal{I}_{n, \beta}$ do not depend on $\left\{t_{i}^{\prime}\right\}$ and write

where

$$
\begin{aligned}
\frac{M^{\prime(n)}\left(t_{1}^{\prime}, \ldots, t_{n}^{\prime}\right)}{n ! \mathcal{I}_{n, \beta}\left(W a^{-2 / \beta}\right)} & =\prod_{i=1}^{n} \hat{t}_{i}^{-2 / \beta} \mathcal{J}_{n, \beta}\left(\left\{\hat{t}_{i}\right\}\right) \\
& =\frac{1}{n} \int_{[0,1]^{n-1}} R\left(\left\{v_{i}\right\}\right) Q\left(\left\{\hat{t}_{i}\right\},\left\{\eta_{i}\right\}\right) d v_{i}
\end{aligned}
$$

$$
\begin{gathered}
Q\left(\left\{\hat{t}_{i}\right\},\left\{\eta_{i}\right\}\right)=\frac{\left(1+\sum_{j=1}^{n} \hat{t}_{j}\right)}{\prod_{i=1}^{n}\left(\hat{t}_{i}^{2 / \beta+1}+\hat{t}_{i}^{2 / \beta} \eta_{i}\right)}, \\
R\left(\left\{v_{i}\right\}\right):=\prod_{i=1}^{n-1} v_{i}^{i(2 / \beta+1)-1}\left(1-v_{i}\right)^{2 / \beta},
\end{gathered}
$$

$\left\{\hat{t}_{i}\right\}$ are related to $\left\{t_{i}^{\prime}\right\}$ via (20) and $\left\{\eta_{i}\right\}$ are related to $\left\{v_{i}\right\}$ via (17). Note that only $Q$ depends on $\left\{t_{i}^{\prime}\right\}$, specifically

$$
Q:=Q\left(\left\{t_{i}^{\prime}\right\}\right)=\frac{\gamma^{-b}\left(1-\gamma \sum_{j=1}^{n} t_{j}^{\prime}\right)^{b}}{\prod_{i=1}^{n}\left[t_{i}^{\prime \alpha+1}+\left(\eta_{i} / \gamma\right)\left(1-\gamma \sum_{j=1}^{n} t_{j}^{\prime}\right) t_{i}^{\prime \alpha}\right]} \frac{\gamma \sum_{j=1}^{n} t_{j}^{\prime}}{\left(1-\gamma \sum_{j=1}^{n} t_{j}^{\prime}\right)}
$$

where $\alpha=2 / \beta$ and $b=n(\alpha+1)$.

This can be written as

$$
Q:=\bar{Q}+\sum_{j=1}^{n} h^{(j)} \bar{Q}
$$

where

$$
\bar{Q}\left(\left\{t_{i}^{\prime}\right\}\right):=\bar{Q}=\frac{\gamma^{-b}\left(1-\gamma \sum_{j=1}^{n} t_{j}^{\prime}\right)^{b}}{\prod_{i=1}^{n}\left[t_{i}^{\prime \alpha+1}+\left(\eta_{i} / \gamma\right)\left(1-\gamma \sum_{j=1}^{n} t_{j}^{\prime}\right) t_{i}^{\prime \alpha}\right]}
$$

and

$$
h^{(j)}\left(\left\{t_{i}^{\prime}\right\}\right):=h^{(j)}=\frac{\gamma t_{j}^{\prime}}{\left(1-\gamma \sum_{i=1}^{n} t_{i}^{\prime}\right)} .
$$

Remark 27. Calculating the partial derivatives $\frac{\partial^{k}}{\partial t_{1}^{\prime}, \ldots \partial t_{k}^{\prime}}$ of $M^{\prime(n)}\left(t_{1}^{\prime}, \ldots, t_{n}^{\prime}\right)$ reduces to the calculation of these derivatives for $\bar{Q}$ and $h^{(j)} \bar{Q}$. This is a tedious but straightforward task. One can use computer symbolic integration (for example, Maple) to easily obtain explicit expressions for these derivatives. Due to the space constraint we will not develop here explicit expressions for the general case $k \leq n$ but only for $k=2$ and arbitrary $n \geq 2$. These expressions are used in the numerical examples presented in this paper.

1) Second-order derivatives: Assume $k=2$ and $n \geq 2$. Let us introduce the auxiliary functions

$$
\begin{aligned}
& A:=\gamma^{-b}\left(1-\gamma \sum_{j=1}^{n} t_{j}^{\prime}\right)^{b} \\
& B:=\prod_{i=1}^{n}\left[t_{i}^{\prime \alpha+1}+\left(\eta_{i} / \gamma\right)\left(1-\gamma \sum_{j=1}^{n} t_{j}^{\prime}\right) t_{i}^{\prime \alpha}\right]^{-1}
\end{aligned}
$$

write $\bar{Q}=A B$, and adopt a subscript notation to denote partial derivatives $\frac{\partial^{2}}{\partial t_{1}^{\prime}, \partial_{2}^{\prime}}$ such that

and

$$
\bar{Q}_{12}:=\frac{\partial^{2} \bar{Q}}{\partial t_{1}^{\prime} t_{2}^{\prime}}=A_{12} B+A_{1} B_{2}+A_{2} B_{1}+A B_{12},
$$

$$
\left[h^{(j)} \bar{Q}\right]_{12}:=\frac{\partial^{2}\left[h^{(j)} \bar{Q}\right]}{\partial t_{1}^{\prime} t_{2}^{\prime}}=h_{12}^{(j)} \bar{Q}+h_{1}^{(j)} \bar{Q}_{2}+h_{2}^{(j)} \bar{Q}_{1}+h^{(j)} \bar{Q}_{12}
$$

where

$$
\bar{Q}_{1}=A_{1} B+A B_{1}, \quad \bar{Q}_{2}=A_{2} B+A B_{2} .
$$

Now only the partial derivatives of the functions $h^{(j)}, A$ and $B$ remain to be calculated, which we do in what follows. 
a) Derivatives of $h^{(j)}$ : For $j=1$ or $2, h^{(j)}$ has the first order derivative

$$
h_{j}^{(j)}=\frac{\gamma\left[1-\gamma\left(\sum_{i=1}^{n} t_{i}^{\prime}-t_{j}^{\prime}\right)\right]}{\left(1-\gamma \sum_{i=1}^{n} t_{i}^{\prime}\right)^{2}}
$$

and the second order derivative

$$
h_{12}^{(j)}=\frac{-\gamma^{2}+2 \gamma^{2}\left[1-\gamma\left(\sum_{i=1}^{n} t_{i}^{\prime}-t_{j}^{\prime}\right)\right]\left(1-\gamma \sum_{i=1}^{n} t_{i}^{\prime}\right)^{-1}}{\left(1-\gamma \sum_{i=1}^{n} t_{i}^{\prime}\right)^{2}}
$$

For $k>2$ and $j=1$ or 2 , the first order derivative is

$$
h_{j}^{(k)}=\frac{\gamma^{2}\left(t_{k}^{\prime}\right)}{\left(1-\gamma \sum_{i=1}^{n} t_{i}^{\prime}\right)^{2}}
$$

while the second order derivative is

$$
h_{12}^{(k)}=\frac{2 \gamma^{3}\left(t_{k}^{\prime}\right)}{\left(1-\gamma \sum_{i=1}^{n} t_{i}^{\prime 3}\right)}
$$

b) Derivatives of $A$ : For the function $A$, the partial derivatives are immediately given

$$
\begin{gathered}
A_{1}=A_{2}=-b \gamma^{-b+1}\left(1-\gamma \sum_{j=1}^{n} t_{j}^{\prime}\right)^{b-1} \\
A_{12}=b(b-1) \gamma^{-b+2}\left(1-\gamma \sum_{j=1}^{n} t_{j}^{\prime}\right)^{b-2} .
\end{gathered}
$$

c) Derivatives of $B$ : The complexity of $B$ requires a couple of extra steps motivating further auxiliary functions

$$
C^{(n)}:=\prod_{j=3}^{n} B^{(j)}
$$

where

$$
B^{(j)}=\left[D^{(j)}-\eta_{j} t_{j}^{\prime \alpha}\left(t_{1}^{\prime}+t_{2}^{\prime}\right)\right]^{-1}
$$

and

$$
D^{(j)}=t_{j}^{\alpha+1}+\left(\eta_{j} / \gamma\right)\left(1-\gamma \sum_{i=3}^{n} t_{i}^{\prime}\right) t_{j}^{\alpha},
$$

while noting that $D^{(1)}$ and $D^{(2)}$ are respectively independent of $t_{2}^{\prime}$ and $t_{1}^{\prime}$. One can write $B=B^{(1)} B^{(2)} C^{(n)}($ for $n=2$, the empty product implies $C^{(2)}=1$ ), hence the partial derivative is given by

$$
\begin{aligned}
B_{12}= & B_{12}^{(1)} B^{(2)} C^{(n)}+B_{1}^{(1)} B_{2}^{(2)} C^{(n)}+B_{2}^{(1)} B_{1}^{(2)} C^{(n)} \\
& +B^{(1)} B_{12}^{(2)} C^{(n)}+B^{(1)} B_{1}^{(2)} C_{2}^{(n)}+B^{(1)} B_{2}^{(2)} C_{1}^{(n)} \\
& +B_{1}^{(1)} B^{(2)} C_{2}^{(n)}+B_{2}^{(1)} B^{(2)} C_{1}^{(n)}+B^{(1)} B^{(2)} C_{12}^{(n)} .
\end{aligned}
$$

The first order derivatives are given by

$$
\begin{aligned}
& B_{1}^{(1)}=-\left(D_{1}^{(1)}-\eta_{1}\left[(\alpha+1) t_{1}^{\prime \alpha}+\alpha t_{1}^{\prime \alpha-1} t_{2}\right]\right)\left(B^{(1)}\right)^{2} \\
& B_{2}^{(2)}=-\left(D_{2}^{(2)}-\eta_{2}\left[(\alpha+1) t_{2}^{\prime \alpha}+\alpha t_{2}^{\prime \alpha-1} t_{1}\right]\right)\left(B^{(2)}\right)^{2}
\end{aligned}
$$

where for $j=1$ or 2 ,

$$
D_{j}^{(j)}=(\alpha+1) t_{j}^{\prime \alpha}+\left(\eta_{j} / \gamma\right) \alpha t_{j}^{\prime \alpha-1}\left(1-\gamma \sum_{i=3}^{n} t_{i}^{\prime}\right)
$$

Furthermore,

$$
\begin{aligned}
& B_{2}^{(1)}=\eta_{1} \alpha t_{1}^{\prime \alpha}\left(B^{(1)}\right)^{2} \\
& B_{1}^{(2)}=\eta_{2} \alpha t_{2}^{\prime \alpha}\left(B^{(2)}\right)^{2} .
\end{aligned}
$$

The second order derivatives are

$$
\begin{aligned}
& B_{12}^{(1)}=\alpha \eta_{1} t_{1}^{\alpha-1}\left(B^{(1)}\right)^{2}-2 \eta_{1} t_{1}^{\alpha \alpha}\left(D_{1}^{(1)}-\eta_{1}\left[(\alpha+1) t_{1}^{\prime \alpha}+\alpha t_{1}^{\prime \alpha-1} t_{2}\right]\right)\left(B^{(1)}\right)^{3} \\
& B_{12}^{(2)}=\alpha \eta_{2} t_{2}^{\alpha-1}\left(B^{(2)}\right)^{2}-2 \eta_{2} t_{2}^{\prime \alpha}\left(D_{2}^{(2)}-\eta_{2}\left[(\alpha+1) t_{2}^{\prime \alpha}+\alpha t_{2}^{\prime \alpha-1} t_{1}\right]\right)\left(B^{(2)}\right)^{3}
\end{aligned}
$$


The product function $C^{(n)}$ has identical first derivatives

$$
C_{1}^{(n)}=C_{2}^{(n)}=C^{(n)} \sum_{i=3}^{n} \eta_{i} t_{i}^{\prime \alpha} B^{(i)}
$$

while

$$
C_{12}^{(n)}=C_{2}^{(n)} \sum_{i=3}^{n} \eta_{i} t_{i}^{\prime \alpha} B^{(i)}+C^{(n)} \sum_{i=3}^{n}\left[\eta_{i} t_{i}^{\prime \alpha} B^{(i)}\right]^{2}
$$

\section{Matrix form of $M^{\prime(n)}$}

Factorial moment measures $M^{\prime(n)}$ admit some matrix-determinant representation. ${ }^{11}$ We introduce some notation. For $n \geq 1$, denote by $\Delta(\boldsymbol{z})$ the $n \times n$ diagonal matrix with entries $\boldsymbol{z}=\left(z_{1}, \ldots, z_{n}\right) \in \mathbb{R}^{n}$ on the diagonal, by $\mathbf{1}=(1, \ldots, 1)^{T}$ the $n$ dimensional column vector of 1's $\left(()^{T}\right.$ stands for the matrix transpose). Denote by $\mathbb{I}=\Delta(\mathbf{1})$ the $n \times n$ identity matrix. We will use " $\times$ " symbol for the matrix multiplication and $|\cdot|$ or $\operatorname{det}()$ for the (square) matrix determinant.

The following identity will be used in what follows (cf [53, Lemma 1.1] or [54, Theorem 13.7.3]). For $n \times n$ matrix $\mathbb{A}$, $n$-dimensional column vector $\mathbf{x}$ and the row vector $\boldsymbol{z}$

$$
|\mathbb{A}+\mathbf{x} \boldsymbol{z}|=|\mathbb{A}|\left(1+\boldsymbol{z} \mathbb{A}^{-1} \mathbf{x}\right) .
$$

For given functions $\varphi, \psi$ from $\mathbb{R}^{+} \times[0,1]$ to $\mathbb{R}^{+}$and two vectors $\mathbf{z}=\left(z_{1}, \ldots, z_{n}\right) \in \mathbb{R}^{n}, \boldsymbol{\eta}=\left(\eta_{1}, \ldots, \eta_{n}\right) \in[0,1]$ denote

$$
\mathbb{K}[\varphi, \psi](\boldsymbol{z}, \boldsymbol{\eta})=\Delta\left(\left(\varphi\left(z_{1}, \eta_{1}\right), \ldots, \varphi\left(z_{n}, \eta_{n}\right)\right)+\mathbf{1} \times\left(\left(\psi\left(z_{1}, \eta_{1}\right), \ldots, \psi\left(z_{n}, \eta_{n}\right)\right) .\right.\right.
$$

Applying the determinant identity (82) we obtain

$$
\operatorname{det}(\mathbb{K}[\varphi, \psi](\boldsymbol{z}, \boldsymbol{\eta}))=\prod_{i=1}^{n} \varphi\left(z_{i}, \eta_{i}\right)\left(1+\sum_{i=1}^{n} \frac{\varphi\left(z_{i}, \eta_{i}\right)}{\psi\left(z_{i}, \eta_{i}\right)}\right)
$$

Note that different rows of the matrix $\mathbb{K}[\varphi, \psi](\boldsymbol{z}, \boldsymbol{\eta})$ depend on different entries of $\boldsymbol{z}$ and consequently by the Leibniz formula for the determinant

$$
\frac{\partial^{n} \operatorname{det}(\mathbb{K}[\varphi, \psi](\boldsymbol{z}, \boldsymbol{\eta}))}{\partial z_{1} \ldots \partial z_{n}}=\operatorname{det}\left(\mathbb{K}\left[\varphi^{\prime}, \psi^{\prime}\right](\boldsymbol{z}, \boldsymbol{\eta})\right),
$$

where $\varphi^{\prime}=\varphi^{\prime}(z, \eta)=\frac{d}{d z} \varphi(z, \eta)$ and similarly for $\psi^{\prime}$. Applying again the identity (82) we obtain

$$
\begin{aligned}
\frac{\partial^{n} \operatorname{det}(\mathbb{K}[\varphi, \psi](\boldsymbol{z}, \boldsymbol{\eta}))}{\partial z_{1} \ldots \partial z_{n}} & =\prod_{i=1}^{n} \varphi^{\prime}\left(z_{i}, \eta_{i}\right)\left(1+\sum_{i=1}^{n} \frac{\varphi^{\prime}\left(z_{i}, \eta_{i}\right)}{\psi^{\prime}\left(z_{i}, \eta_{i}\right)}\right) .
\end{aligned}
$$

Define the following two functions on $\mathbb{R}^{+}$and $\mathbb{R} \times[0,1]$, respectively,

$$
G(z)=z^{-1}, \quad H(z, \eta)=z^{-2 / \beta}(z+\eta)^{-1} .
$$

Remark 28. Using the determinant identity (82) and (80) it is easy to see that

$$
Q\left(\left\{\hat{t}_{i}\right\},\left\{\eta_{i}\right\}\right)=\operatorname{det}\left(\mathbb{K}\left[H, \frac{H}{G}\right](\hat{\boldsymbol{t}}, \boldsymbol{\eta})\right) .
$$

The above representation may offer an alternative way of calculating the partial derivatives of $Q$, following the lines explained in [54, Sections 15.8-15.9] and using the Sherman-Morrison formula; [54, eq. (2.25)]).

\section{E. Proof of Lemma 11}

By the displacement theorem [40, Section 1.3.3] and Campbell's theorem [40, Corolloary 2.2], $\Psi$ is a Poisson point process with intensity measure

$$
\begin{aligned}
\Lambda(s, t) & =\mathbf{E}\left[\sum_{(Y, T) \in \widetilde{\Theta}} \mathbb{1}(Y \leq s, T \leq t)\right] \\
& =\lambda \mathbf{E} \int_{\mathbb{R}^{2}} \mathbb{1}(Y \leq s) \mathbb{1}(T \leq t) d x \\
& =\lambda(2 \pi) \mathbf{E} \int_{0}^{\infty} \mathbb{1}\left(r \leq(s P S)^{1 / \beta}\right) \mathbb{1}(T \leq t) r d r .
\end{aligned}
$$

\footnotetext{
${ }^{11}$ This representation is different from that used to define determinantal point processes, which suggests, but does not prove, that the STINR process is not a determinantal point process.
} 


\section{F. Schuette-Nesbitt formula}

The following result is often used in insurance mathematics (see, for example, [42] for a proof). Let $B_{1}, B_{2}, \ldots B_{m}$ denote arbitrary events, and define the $n$th symmetric sum for them as $\mathcal{S}_{n}=\sum_{\substack{i_{1}, i_{2}, \ldots, i_{n} \\ \text { disininet }}} \mathbf{P}\left(\bigcap_{j=1}^{n} B_{i_{j}}\right)$. Let the random variable $\mathcal{N}$ denote the number of the aforementioned events that occur, that is $\mathcal{N}=\sum_{i=1}^{m} \mathbb{1}_{B_{i}}$.

Theorem 29. [Schuette-Nesbitt formula] For arbitrary coefficients $c_{1}, c_{2}, \ldots c_{m}$ the following holds true

$$
\sum_{n=0}^{m} c_{n} \mathbf{P}(\mathcal{N}=n)=\sum_{n=0}^{m}\left(\Delta^{n} c\right)_{0} \mathcal{S}_{n}
$$

where $\Delta$ is the forward difference operator; that is, $(\Delta c)_{k}=c_{k+1}-c_{k}$ and $\Delta^{k}=\Delta \Delta^{k-1}$.

\section{REFERENCES}

[1] J. Andrews, F. Baccelli, and R. Ganti, "A tractable approach to coverage and rate in cellular networks," IEEE Trans. Commun., vol. 59, no. 11, pp. $3122-3134$, november 2011.

[2] B. Błaszczyszyn, M. K. Karray, and H. P. Keeler, "Using Poisson processes to model lattice cellular networks," in INFOCOM, 2013 Proceedings IEEE, 2013, pp. 773-781.

[3] — - "Wireless networks appear Poissonian due to strong shadowing," arxiv:1409.4739, 2014.

[4] T. X. Brown, "Cellular performance bounds via shotgun cellular systems," Selected Areas in Communications, IEEE Journal on, vol. 18, no. 11, pp. 2443-2455, 2000.

[5] B. Błaszczyszyn, "Factorial-moment expansion for stochastic systems," Stoch. Proc. Appl., vol. 56, pp. 321-335, 1995.

[6] B. Błaszczyszyn, E. Merzbach, and V. Schmidt, "A note on expansions for functional of spatial marked processes," Statist. and Probab. Lett., vol. 36, pp. 299-306, 1997.

[7] B. Błaszczyszyn and H. Keeler, "Equivalence and comparison of heterogeneous cellular networks," in Proc. of WDNCN2013, 2013.

[8] E. N. Gilbert and H. O. Pollak, "Amplitude distribution of shot noise," Bell Systems Technical Journal, vol. 39, pp. 333-350, 1960.

[9] S. Lowen and M. C. Teich, "Power-law shot noise," Information Theory, IEEE Transactions on, vol. 36, no. 6, pp. $1302-1318,1990$.

[10] M. Haenggi, "A geometric interpretation of fading in wireless networks: Theory and applications," Information Theory, IEEE Transactions on, vol. 54, no. 12, pp. 5500-5510, 2008.

[11] B. Błaszczyszyn, M. Karray, F. Klepper et al., "Impact of the geometry, path-loss exponent and random shadowing on the mean interference factor in wireless cellular networks," in Third Joint IFIP Wireless and Mobile Networking Conference (WMNC), 2010.

[12] P. Pinto, J. Barros, and M. Win, "Secure communication in stochastic wireless networks - Part I: Connectivity," Information Forensics and Security, IEEE Transactions on, vol. 7, no. 1, pp. 125-138, 2012.

[13] H. S. Dhillon, R. K. Ganti, and J. G. Andrews, "A tractable framework for coverage and outage in heterogeneous cellular networks," in Information Theory and Applications Workshop (ITA), 2011. IEEE, 2011, pp. 1-6.

[14] H. Dhillon, R. Ganti, F. Baccelli, and J. Andrews, "Modeling and analysis of K-tier downlink heterogeneous cellular networks," IEEE J. Sel. Areas Commun., vol. 30, no. 3, pp. 550-560, april 2012.

[15] P. Madhusudhanan, J. Restrepo, Y. Liu, T. Brown, and K. Baker, "Multi-tier network performance analysis using a shotgun cellular system," in Global Telecommunications Conference (GLOBECOM 2011), 2011 IEEE, 2011, pp. 1-6.

[16] P. Madhusudhanan, J. Restrepo, Y. Liu, and T. Brown, "Downlink coverage analysis in a heterogeneous cellular network," in Global Communications Conference (GLOBECOM), 2012 IEEE, 2012, pp. 4170-4175.

[17] S. Mukherjee, "Downlink SINR distribution in a heterogeneous cellular wireless network with max-SINR connectivity," in Communication, Control, and Computing (Allerton), 2011 49th Annual Allerton Conference on. IEEE, 2011, pp. 1649-1656.

[18] H. Keeler, B. Blaszczyszyn, and M. Karray, "SINR-based $k$-coverage probability in cellular networks with arbitrary shadowing," in Information Theory Proceedings (ISIT), 2013 IEEE International Symposium on, 2013, pp. 1167-1171.

[19] S. Mukherjee, "Distribution of downlink SINR in heterogeneous cellular networks," Selected Areas in Communications, IEEE Journal on, vol. 30, no. 3, pp. 575-585, 2012.

[20] H.-S. Jo, Y. J. Sang, P. Xia, and J. Andrews, "Heterogeneous cellular networks with flexible cell association: A comprehensive downlink SINR analysis," Wireless Communications, IEEE Transactions on, vol. 11, no. 10, pp. 3484 3495, 2012.

[21] T. T. Vu, L. Decreusefond, and P. Martins, "An analytical model for evaluating outage and handover probability of cellular wireless networks," in Proc of WPMC, 2012, pp. 643-647.

[22] S. Mukherjee, "Downlink SINR distribution in a heterogeneous cellular wireless network with biased cell association," in Communications (ICC), 2012 IEEE International Conference on. IEEE, 2012, pp. 6780-6786. 
[23] A. Giovanidis and F. Baccelli, "A stochastic geometry framework for analyzing pairwise-cooperative cellular networks," arXiv preprint arXiv:1305.6254, 2013.

[24] S. Akoum and R. W. Heath, "Limited feedback for temporally correlated MIMO channels with other cell interference," Signal Processing, IEEE Transactions on, vol. 58, no. 10, pp. 5219-5232, 2010.

[25] X. Zhang and M. Haenggi, "The performance of successive interference cancellation in random wireless networks," in Global Communications Conference (GLOBECOM), 2012 IEEE. IEEE, 2012, pp. 2317-2321.

[26] — - "On decoding the $k$ th strongest user in Poisson networks with arbitrary fading distribution," in 47th Asilomar Conference of Signals, Systems and Computers (Asilomar'13), Pacific Grove, CA, Nov. 2013.

[27] — "Successive Interference Cancellation in Downlink Heterogeneous Cellular Networks," in IEEE Global Communications Conference (GLOBECOM-HetSNets'13), Atlanta, GA, 2013.

[28] M. Wildemeersch, T. Q. Quek, M. Kountouris, A. Rabbachin, and C. H. Slump, "Successive interference cancellation in heterogeneous cellular networks," arXiv preprint arXiv:1309.6788, 2013.

[29] D. J. Daley and D. Vere-Jones, An introduction to the theory of point processes. Vol. I, 2nd ed., ser. Probability and its Applications (New York). New York: Springer, 2003.

[30] —, An introduction to the theory of point processes. Vol. II, 2nd ed., ser. Probability and its Applications (New York). New York: Springer, 2008.

[31] D. Stoyan, W. Kendall, and J. Mecke, Stochastic Geometry and its Applications, 2nd ed. Wiley, 1995.

[32] D. P. Kroese and V. Schmidt, "Light-traffic analysis for queues with spatially distributed arrivals," Mathematics of Operations Research, vol. 21, no. 1, pp. pp. 135-157, 1996.

[33] R. Ganti, F. Baccelli, and J. Andrews, "Series expansion for interference in wireless networks," Information Theory, IEEE Transactions on, vol. 58, no. 4, pp. 2194-2205, 2012.

[34] H. P. Keeler and B. Błaszczyszyn, "SINR in wireless networks and the two-parameter Poisson-Dirichlet process," IEEE Wireless Comm Letters, vol. 3, no. 5, 2014.

[35] J. Pitman and M. Yor, "The two-parameter Poisson-Dirichlet distribution derived from a stable subordinator," The Annals of Probability, vol. 25, no. 2, pp. 855-900, 1997.

[36] K. Handa, "The two-parameter Poisson-Dirichlet point process," Bernoulli, vol. 15, no. 4, pp. 1082-1116, 2009.

[37] F. Gunnarsson, "Power control in wireless networks: characteristics and fundamentals," in Wireless communications systems and networks, M. Guizani, Ed., 2004, pp. 179-208.

[38] D. Panchenko, The Sherrington-Kirkpatrick model. Springer, 2013.

[39] J. F. C. Kingman, Poisson Processes, 1st ed. Oxford University Press, 1993.

[40] F. Baccelli and B. Błaszczyszyn, Stochastic Geometry and Wireless Networks, Volume I - Theory, ser. Foundations and Trends in Networking. NoW Publishers, 2009, vol. 3, No 3-4.

[41] W. Feller, An Introduction to Probability Theory and its Applications, vol I., 3rd ed. New York: J. Wiley \& Sons, 1968.

[42] H. U. Gerber, Life Insurance Mathematics. Springer, 1995.

[43] S. P. Weber, J. G. Andrews, X. Yang, and G. De Veciana, "Transmission capacity of wireless ad-hoc networks with successive interference cancellation," Information Theory, IEEE Transactions on, vol. 53, no. 8, pp. 2799-2814, 2007.

[44] Y. Bar-Ness and H. Bunin, "Adaptive co-channel interference cancellation and signal separation method," in Communications, 1989. ICC'89, BOSTONICC/89. Conference record.'World Prosperity Through Communications', IEEE International Conference on. IEEE, 1989, pp. 825-830.

[45] W. Xu and S. Sezginer, "Co-channel interference cancellation in reuse-1 deployments of wimax system," in Wireless Communications and Networking Conference (WCNC), 2012 IEEE. IEEE, 2012, pp. 342-346.

[46] H. P. Keeler, "Studying the SINR process in Poisson networks by using its factorial moment measures," MATLAB Central File Exchange, 2014, available at http://www.mathworks.com.au/matlabcentral/fileexchange/45299-studying-thesinr-process-in-poisson-networks-by-using-its-factorial-moment-measures.

[47] F. Kuo and I. Sloan, "Lifting the curse of dimensionality," Notices of the AMS, vol. 52, no. 11, pp. 1320-1328, 2005.

[48] J. Dick, F. Y. Kuo, and I. H. Sloan, "High-dimensional integration-the Quasi-Monte Carlo way," Acta Numerica, vol. 22, pp. 133-288, 2013.

[49] G. L. Stüber, Principles of Mobile Communication, 2nd ed. NYC, NY, USA: Springer, 2011.

[50] N. Miyoshi and T. Shirai, "A cellular network model with Ginibre configurated base stations," Adv. Appl. Probab., vol. 46, no. 3, pp. 832-845, 2014

[51] I. Nakata and N. Miyoshi, "Spatial stochastic models for analysis of heterogeneous cellular networks with repulsively deployed base stations," Performance Evaluation, vol. 78, pp. 7-17, 2014.

[52] (2012, Accessed on the 10th of September) Digital Library of Mathematical Functions. National Institute of Standards and Technology. Release 1.0.5 of 2012-10-01. [Online]. Available: http://dlmf.nist.gov/

[53] J. Ding and A. Zhou, "Eigenvalues of rank-one updated matrices with some applications," Applied Mathematics Letters, vol. 20, no. 12, pp. 1223-1226, 2007.

[54] D. A. Harville, Matrix algebra from a statistician's perspective. Springer, 2008. 